\title{
Synaptic Vesicle Dynamics in Living Cultured Hippocampal Neurons Visualized with CY3-Conjugated Antibodies Directed against the Lumenal Domain of Synaptotagmin
}

\author{
Kajetan Kraszewski,, Olaf Mundigl, ${ }^{1}$ Laurie Daniell,' Claudia Verderio, ${ }^{2}$ Michela Matteoli, ${ }^{2}$ and \\ Pietro De Camilli' \\ 'Department of Cell Biology and Howard Hughes Medical Institute, Yale University School Medicine, New Haven, \\ Connecticut 06510 and ${ }^{2} \mathrm{CNR}$ Center of Cytopharmacology and Department of Medical Pharmacology, University of \\ Milano, Milano, Italy
}

\begin{abstract}
Antibodies directed against the lumenal domain of synaptotagmin I conjugated to $\mathrm{CY}_{3}$ (CY3-Syt,-Abs) and video microscopy were used to study the dynamics of synaptic vesicles in cultured hippocampal neurons. When applied to cultures after synapse formation, CY3-Syt,-Abs produced a strong labeling of presynaptic vesicle clusters which was markedly increased by membrane depolarization. The increase of the rate of CY3-Syt-Ab uptake in a high $\mathrm{K}^{+}$medium was maximal during the first few minutes but persisted for as long as $60 \mathrm{~min}$. In axons developing in isolation, CY3-Syt,-Abs, in combination with electron microscopy immunocytochemistry, revealed the presence of synaptic vesicle clusters which move in bulk in anterograde and retrograde direction. Clusters are present both in the axon shaft and in filopodia but not in the filopodia of the growth cone. Both presynaptic vesicle clusters and clusters present in isolated axons were disrupted by okadaic acid as previously shown for synaptic vesicle clusters at the frog neuromuscular junction. These findings indicate that synaptic vesicle aggregation may occur independently of cell-cell interaction, but that, in the absence of a synaptic contact, vesicle clusters are not stably anchored to a given region of the cell surface. Labeling of synaptic vesicles in immature isolated neurons was found to be depolarization and $\mathrm{Ca}^{2+}$ dependent, demonstrating that $\mathrm{Ca}^{2+-}$ regulated exocytosis is an intrinsic characteristic of synaptic vesicles irrespective of their localization at a synapse.
\end{abstract}

[Key words: growth cones, exocytosis, endocytosis, synaptogenesis, synapse]

Synaptic vesicles (SVs) are highly specialized secretory organelles by which neurons secrete "fast" neurotransmitters at synapses. In mature neurons SVs are clustered in axonal varicosities

\footnotetext{
Received Dec. 7, 1994; revised Feb. 6, 1995; accepted Feb. 8, 1995.

We thank Dr. Watt Webb for discussion, Dr. Thiery Galli for critical review of the manuscript, Dr. Reinhard Jahn for the gift of Syt $t_{c}-\mathrm{Ab}$, Dr. Heiner Niemann for the gift of clostridial neurotoxins, and Henry Tan for photographic assistance. This work was supported in part by NIH Grants (CA46128 and DK43078) (P.D.C.), by a Brown-Coxe postdoctoral fellowship (K.K), by a NATO collaborative grant (P.D.C., M.M.), and by the Italian National Research Council (M.M.).

Correspondence should be addressed to Pietro De Camilli, Department of Cell Biology, Boyer Center for Molecular Medicine, 295 Congress Avenue, New Haven, CT 06510.

Copyright (C) 1995 Society for Neuroscience $0270-6474 / 95 / 154328-15 \$ 05.00 / 0$
}

which represent the presynaptic elements of synapses. They undergo exocytosis selectively at specialized regions of the presynaptic plasmalemma called active zones and their rate of exocytosis is dramatically stimulated by depolarization-induced $\mathrm{Ca}^{2+}$ influx (De Camilli and Jahn, 1990; Jessel and Kandel, 1992; Südhof et al., 1993; Bennett and Scheller, 1994).

Until recently, the properties of SVs in situ could only be studied by conventional morphological approaches which involve cell fixation, or by using electrophysiological techniques which detect effects produced by neurotransmitter release. New probes have now been developed which make possible to monitor morphologically, at the light microscopic level, SV dynamics in the living cell. The best characterized of these probes is the styryl dye FM1-43, which binds with fast "on" and "off" rates to phospholipid bilayers. This dye labels very effectively endocytic vesicles and its rapid dissociation from membranes allows to monitor reexposure of such vesicles to the cell surface by exocytosis (Betz and Bewick, 1992; Betz and Bewick, 1993). At presynaptic sites, where exo-endocytotic recycling is accounted for primarily by SV membranes, exo-exocytosis of these organelles can be monitored by studying the loading, or unloading, of the dye into and from the nerve terminal (Betz and Bewick, 1992; Betz et al., 1992a; Ryan et al., 1993).

Other potential probes for the study of SVs in vivo are antibodies directed against the lumenal domain of SV-specific proteins. One such antibody, directed against the lumenal N-terminus of synaptotagmin I, was previously shown by us to be internalized via SVs (Matteoli et al., 1992; Mundigl et al., 1993) and to recycle in parallel with the vesicle. These studies, in which the visualization of the internalized antibodies was performed on fixed cells, raised the possibility that a fluorescent probe based on antibodies may be used for studies of SVs in the living neuron. While one can anticipate that an antibodybased probe may be less effective in monitoring with close temporal fidelity a fast process such as SV exocytosis, antibodies could offer in principle other advantages. One advantage is the very high specificity of SV labeling which contrasts with the property of FM1-43 to labels nonspecifically all endocytic membranes (Betz and Bewick, 1992). Thus, antibodies could be used to monitor SV exo endocytosis at sites, for example, developing isolated axons, where it is not known how predominant SV recycling is over other types of recycling. Another advantage is the stable association between antibodies and the lumen of the vesicles, which makes possible to monitor vesicle traffic over 
long time scales, irrespective of whether the vesicle undergoes new rounds of exocytosis.

We report here the characterization of antibodies directed against the lumenal domain of synaptotagmin I and directly conjugated to the fluorochrome CY3 (CY3-Syt $-\mathrm{Abs}$ ) as a "vital" label for SVs. We demonstrate that this label can be used as a quantitative tool to monitor SVs exocytosis and that it provides information complementary to the information which can be obtained with FM1-43. Using this new probe we show that SVs undergo $\mathrm{Ca}^{2+}$ - and depolarization-dependent exocytosis even before synapse formation. We also show that in isolated developing axons SVs are arranged in small highly motile clusters located along the axon shaft as well as in filopodia, but excluded from the filopodia of the growth cone. Thus, the property of forming clusters and to undergo regulated exocytosis is intrinsic to SVs and is not dependent upon the formation of a presynaptic specialization.

\section{Materials and Methods}

Antibodies and materials. Rabbit polyclonal antibodies directed against the lumenal domain of synaptotagmin I were previously described (Mundigl et al., 1993). Mouse monoclonal antibodies directed against the cytoplasmic domain of synaptotagmin I were kind gift of Dr. R. Jahn (Yale University, New Haven, CT) (Brose et al., 1992; Mundigl et al., 1993). Secondary antibodies were from Jackson Laboratories (West Grove, PA). Affinity-purified Syt,-Abs were conjugated with CY3 (Biological Detection System, Pittsburgh, PA) as described (Southwick et al., 1990). The average fluorochrome to protein ratio was measured to be $4: 1$. Tetanus toxin and Botulinum toxin D and F were kind gift of H. Niemann (Tübingen). FM1-43 was purchased from Molecular Probes (Eugene, Oregon) and okadaic acid from Sigma (St. Louis, MO).

Hippocampal cultures. Primary cultures of hippocampal neurons were prepared from the hippocampi of $18 \mathrm{~d}$ old fetal rats as described by Bartlett and Banker (1984). Cultures were used after 3 or $8 \mathrm{~d}$ in vitro (DIV).

Cell labeling protocols. The culture medium of neurons was substituted with Krebs-Ringer-HEPES (KRH: $128 \mathrm{~mm} \mathrm{NaCl}, 25 \mathrm{~mm}$ HEPES, $4.8 \mathrm{mM} \mathrm{KCl}, 1.3 \mathrm{mM} \mathrm{CaCl}, 1.2 \mathrm{mM} \mathrm{MgSO}_{4}, 1.2 \mathrm{mM} \mathrm{KH}_{2} / \mathrm{K}_{2} \mathrm{HPO}_{4}$, and $5.6 \%$ glucose). After 10 min KRH was substituted with new KRH containing CY3-Syt $-\mathrm{Abs}$ at the concentration of $3.75 \mu \mathrm{g} / \mathrm{ml}$. Cells were than mounted onto the microscope stage for observation. Okadaic acid $(2 \mu \mathrm{M})$ or FM1-43 (1 $\mu \mathrm{M})$ was added via a superfusion system. For FM143 labeling the protocol described by Ryan et al. (1993) was used. For quantitative analysis of CY3-Syt $-\Lambda b$ uptake, the incubation with these antibodies was performed in the following media: $\mathrm{KRH}, \mathrm{KRH}$-high $\mathrm{K}^{+}$ $\left(57 \mathrm{mM} \mathrm{K}^{+}\right.$and a corresponding reduction in $\mathrm{Na}^{+}$), $\mathrm{KRH}$-zero $\mathrm{Ca}^{2+}$ (with a corresponding increase in $\mathrm{Mg}^{2}$ and $1 \mathrm{~mm}$ EGTA). After variable times, neurons were washed with fresh KRH. Cells were then fixed after the washings with $4 \%$ formaldehyde (freshly prepared from paraformaldehyde) in $0.1 \mathrm{M}$ sodium phosphate buffer containing $0.12 \mathrm{M}$ sucrose. Fixed cells were then permeabilized and reacted with antibod ies to the cytoplasmic domain of synaptotagmin ( $\left.\mathrm{Syt}_{\mathrm{c}}-\mathrm{Abs}\right)$ followed by FITC-conjugated secondary antibody as previously described (Mundigl et al., 1993). In some experiments, cells were pretreated for $1 \mathrm{hr}$ with tetanus of Botulinum toxins in $\mathrm{KRH}$ prior to incubation with CY3-Syt Abs. Unless otherwise stated, all incubations of living neurons were performed at $37^{\circ} \mathrm{C}$.

Electron microscopy. Neurons were fixed and further processed for electron microscopy as previously described (Mundigl et al., 1993). In some experiments fixation was preceded by an incubation of the neurons for $1 \mathrm{hr}$ at $37^{\circ} \mathrm{C}$ in $\mathrm{KRH}$-high $\mathrm{K}^{+}$with horseradish peroxidase (HRP) $(10 \mathrm{mg} / \mathrm{ml})$. HRP reaction product was then developed as described previously (De Camilli et al., 1983). In other experiments fixation was preceded by the series of the following pretreatments all performed in $\mathrm{KRH}$ at $37^{\circ} \mathrm{C}: 1 \mathrm{hr}$ with $\mathrm{Syt}_{1}-\mathrm{Abs}(3.75 \mu \mathrm{g} / \mathrm{ml}), 10 \mathrm{~min}$ wash, $1 \mathrm{hr}$ with colloidal gold-protein A conjugates $(6 \mathrm{~nm}$ prepared as described by Slot and Geuze, 1983). This sequence was aimed at labeling with colloidal gold particles SVs which undergo recycling (Matteoli et al., 1992).

Video microscopy. Neurons on coverslips were mounted in a chamber at room temperature on the stage of a Zeiss Axiovert 35 equipped for
DIC microscopy and for epifluorescence (100 W Hg lamp). Excitation and emission filters, respectively, were $540 \pm 12.5 \mathrm{~nm}$ and $590 \pm 17.5$ $\mathrm{nm}$ (CY3), $480 \pm 15 \mathrm{~nm}$ and $530 \pm 15 \mathrm{~nm}$. (fluorescein and FM143). A chilled CCD camera (Photometrics, Tucson, AZ model CH 250) was used for DIC and quantitative fluorescent measurements. An SIT camera (Hamamatsu Corp., Middlesex, NJ, model C2400) was used for time-lapse fluorescence microscopy. The specimens were observed with a spatial frequency of $0.3 \mu \mathrm{m} / \mathrm{pixel}$ (SIT camera) or $0.1 \mu \mathrm{m} / \mathrm{pixel}$ and $0.07 \mu \mathrm{m} / \mathrm{pixel}$ (CCD camera). For the comparison of FM1-43 and CY3$\mathrm{Syt}_{1}-\mathrm{Ab}$ labeling, cells were first labeled with $\mathrm{CY} 3-\mathrm{Syt}_{1}-\mathrm{Ab}$ and imaged for the CY3 signal. Then cells were superfused with FM1-43 and processed for FM1-43 labeling using the fluorescein channel. Images were acquired and processed with an IBM computer and software (IMAGEl and METAMORPH) from Universal Imaging Corporation (West Chester, PA). Images were stored using an analog, optical disk recorder (Panasonic, model TQ-2028F) or a digital, optical disk driver (Panasonic). Images were printed with a video printer (Toshiba).

Quantitative analysis of fuorescence intensity. For quantitative measurements, images obtained with the CCD camera after background subtraction were used. Ratios between CY3 and fluorescein fluorescence were obtained by determining the average pixel brightness in a rectangle of $10 \times 10$ pixels (corresponding to $1.0 \times 1.0 \mu \mathrm{m}$ ) which overlapped a fluorescent spot. The ratio calculated was typically very similar on different spots within the same neuron. For each experimental condition measurements from at least eight different neurons from at least three experiments were pooled.

Observations of the background fluorescence produced by CY3-Syt Abs absorbed on glass coverslips allowed us to develop a method to estimate the average fluorescence of the smallest unitary element of fluorescence of the conjugates. Glass coverslips were washed in nitric oxide, rinsed in distilled water and incubated with Cy3-Syt - Abs diluted in phosphate buffered saline at room temperature. These coverslips exhibited a dotty pattern of fluorescence when observed at high magnification and for long exposure times $(>10 \mathrm{sec})$ with the CCD camera. The diameter of the dots was about $250 \mathrm{~nm}$ and therefore consistent with a size of the fluorescent particle below the resolution limit of the light microscope. To obtain the intensity of fluorescence of a single dot, we integrated the fluorescent signal from a rectangle of $0.7 \mu \mathrm{m} \times 0.7$ $\mu \mathrm{m}(10 \times 10$ pixels) which encloses the dot and subtracted from this value the integrated fluorescence of a rectangle from a nearby region not containing any fluorescent spot. While the number of dots per unit area increased with the incubation time and with the concentration of CY3-Syt ${ }_{1}$-Abs (Fig. 1a), the fluorescence intensity in a single dot (elementary unit fluorescence measured in arbitrary camera units) was roughly constant and independent of such parameters. An histogram which shows the distribution of the integrated fluorescence of 100 dots observed on two coverslips which had been incubated for either 4 or $20 \mathrm{~min}$ is shown in Figure $1 b$. The histograms clearly show two similar distributions [average camera units: $8.4 \pm 3.2(4 \mathrm{~min}), 8.3 \pm 3.2(20$ min)]. This finding suggests that each spot may represent a single CY3/ IgG conjugate. Consistent with these interpretations, when CY3-IgG conjugates with different average stoichiometric ratios of CY3 to IgG were used, a different average fluorescence intensity of the spots was observed. However, the correspondence of the spots to single IgG conjugates remains to be confirmed by additional studies.

Irrespectively of whether each spot represents a single CY3-Syt $-\mathrm{Ab}$, the average elementary unit fluorescence defined above can be used to obtain an estimate of the minimal number of CY3-Syt - Abs molecules comprised in a single labeled structure in neuronal cultures. To obtain such a number, the fluorescence of the smaller rectangle enclosing the structure was measured. Then the background fluorescence intensity measured on a rectangle taken from a surrounding neuronal region was subtracted from this value. The resulting number was divided by the elementary fluorescent unit. CCD camera exposures for these determination were always of identical length $(10 \mathrm{sec})$.

\section{Results}

In vivo labeling of $S V S$ in neurons after synapse formation

The pattern of labeling produced in 8 DIV neurons by CY3Syt $_{1}$-Abs applied in $\mathrm{KRH}$-high $\mathrm{K}^{+}$medium for $20 \mathrm{~min}$, that is, conditions sufficient to produce a strong CY3 immunofluorescence signal (see below), is shown in Figure $2 a$. The neuron was examined with a CCD camera to generate a fluorescence and a DIC image of the living cell. At this developmental stage 

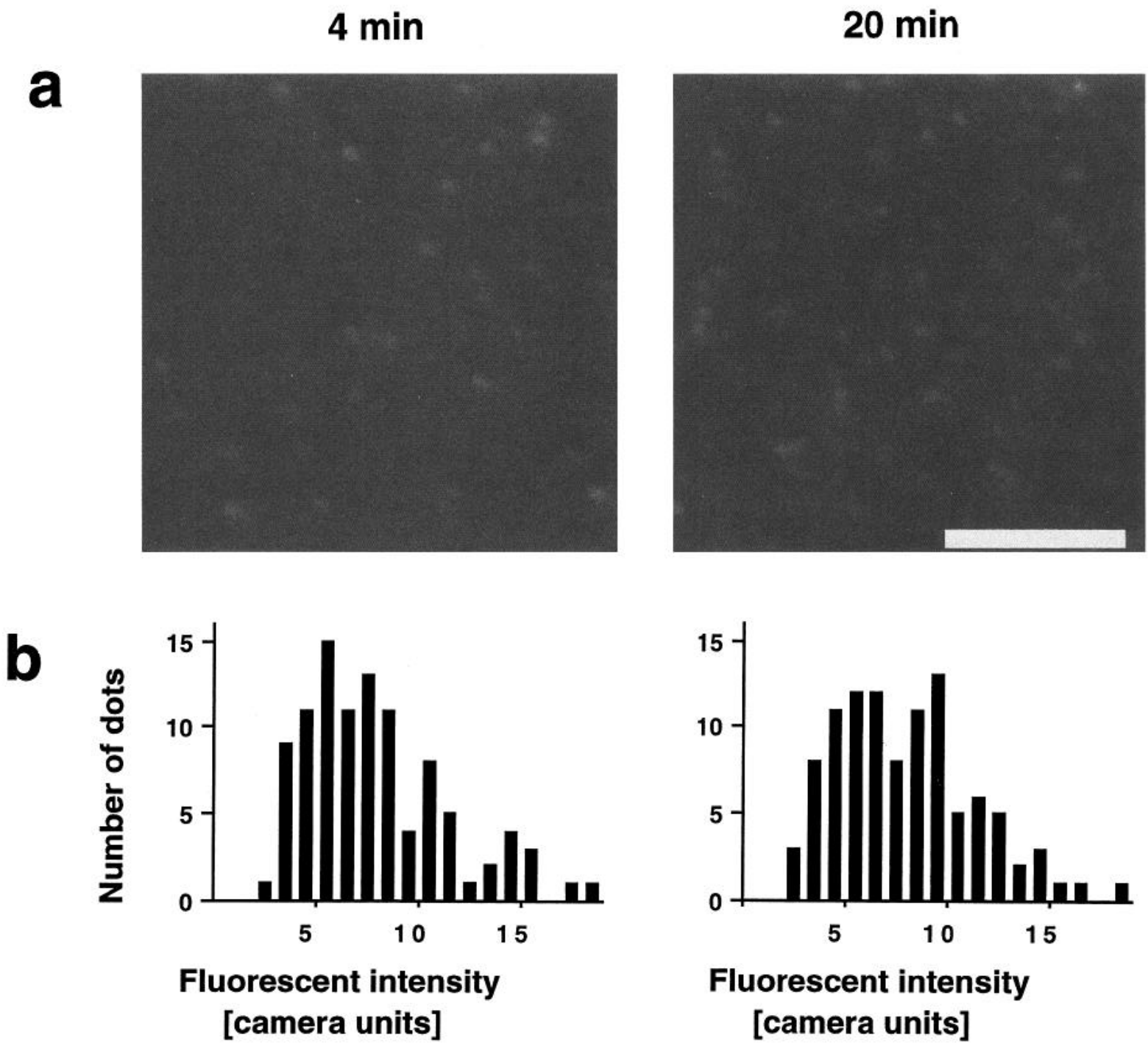

Figure 1. Characterization of elementary fluorescence CY3-Syt,-Abs units. $a$, Dotted pattern of fluorescence detected by a CCD (10 sec exposure) on glass coverslips incubated with CY3-Syt,-Abs for $4 \mathrm{~min}$ and $20 \mathrm{~min}$. The number of fluorescence spots for unit area is higher on the coverslip incubated for the longer time. $b$, Fluorescence intensity distribution of the dots detected by the CCD camera on coverslips after 4 and 20 min incubation with antibodies. Scale bar, $5 \mu \mathrm{m}$.

(stage 5 of Dotti et al., 1988), abundant synaptic contacts have already formed. The DIC image demonstrated the presence of several axons which run along the surface of the perikaryon and dendrites. The most prominent CY3-labeled structures were large patches of bright fluorescence aligned with the profiles of dendrites at sites where axons contact them. These patches precisely coincided with presynaptic clusters of SVs as was demonstrated by fixing the neurons and counterstaining them, after detergent permeabilization, with monoclonal antibodies directed against the cytoplasmic domain of synaptotagmin I or other SV proteins (Fletcher et al., 1991; Matteoli et al., 1992). In addition to the bright dots, fluorescent structures of smaller size and weaker fluorescence were observed throughout the axons including axonal segments which do not form any contacts (see also Fig. 2a). Most of these heterogeneous fluorescent dots in transit along axons may correspond to the endosomes described by Parton et al. (1992).

These interpretations are supported by observations of the motility of fluorescent structures by video microscopy using an SIT camera. Figure $3 a$ shows a low power view of a neuron from a culture which has been exposed to CY3-Syt $-\mathrm{Ab}$ as described for Figure 2. The coexistence of two patterns of immunoreactivity in the axons which surround the neuron is clearly visible. Three time-lapse images of fields 1 and 2, respectively,

Figure 2. Micrographs demonstrating the "in vivo" labeling of SVs by CY3-Syt,-Abs. DIC and fluorescent images were collected with a CCD camera and superimposed. To increase contrast DIC images were printed in green and fluorescent images in orange. Cultures were incubated with CY3-Syt - Abs for $20 \mathrm{~min}$ in KRH-high $\mathrm{K}^{+}, a$, Mature culture rich in synapses (8 DIV). Large labeled structures represent presynaptic SV clusters. Smaller labeled particles are visible in preterminal axons. $b$, Distal segment of an isolated axon from a young culture ( 3 DIV). Labeled particles of variable size and fluorescence intensity are present in the shaft and in filopodia but are absent from the growth cones. Scale bar, $5 \mu \mathrm{m}$. 


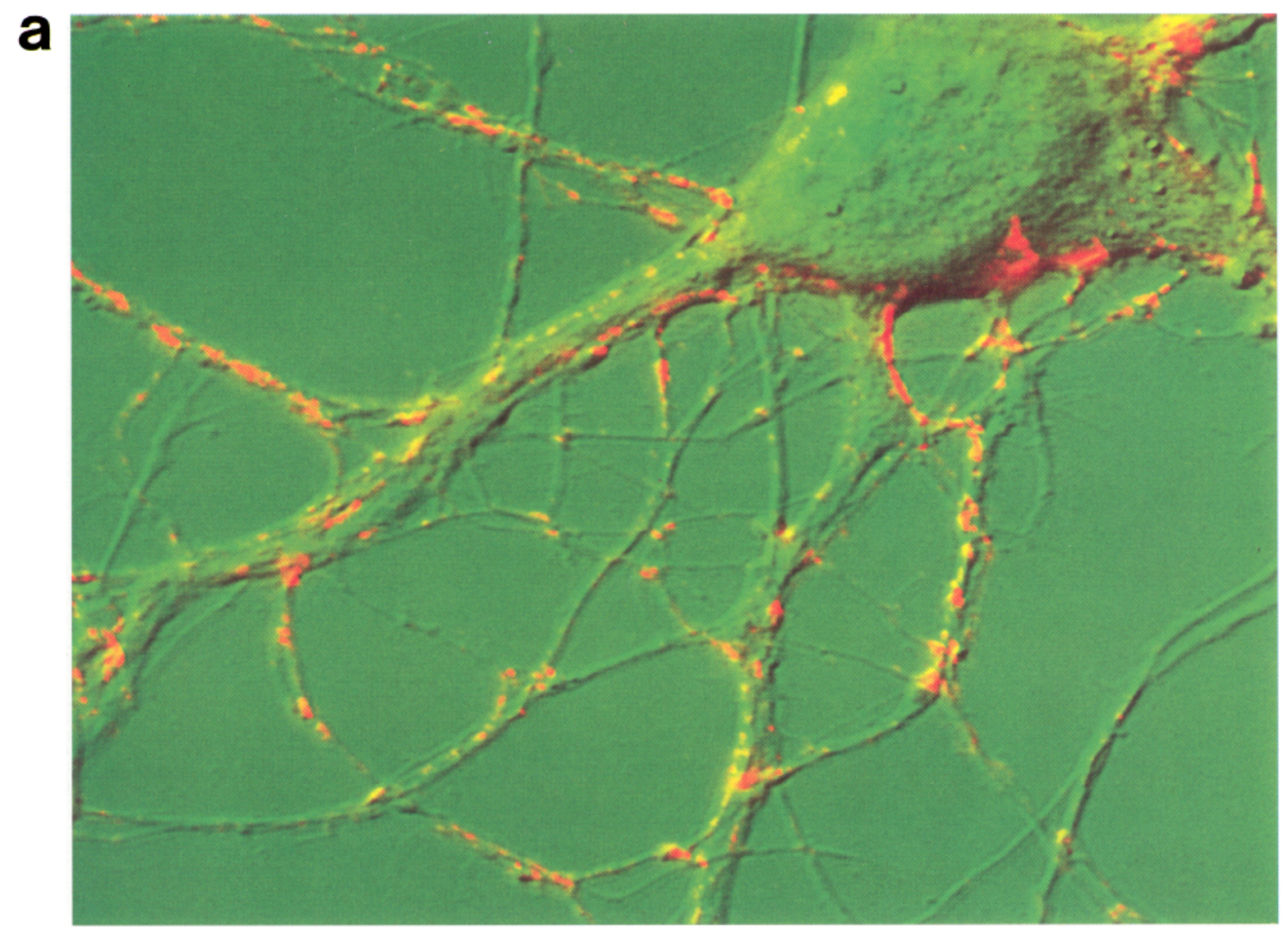

b

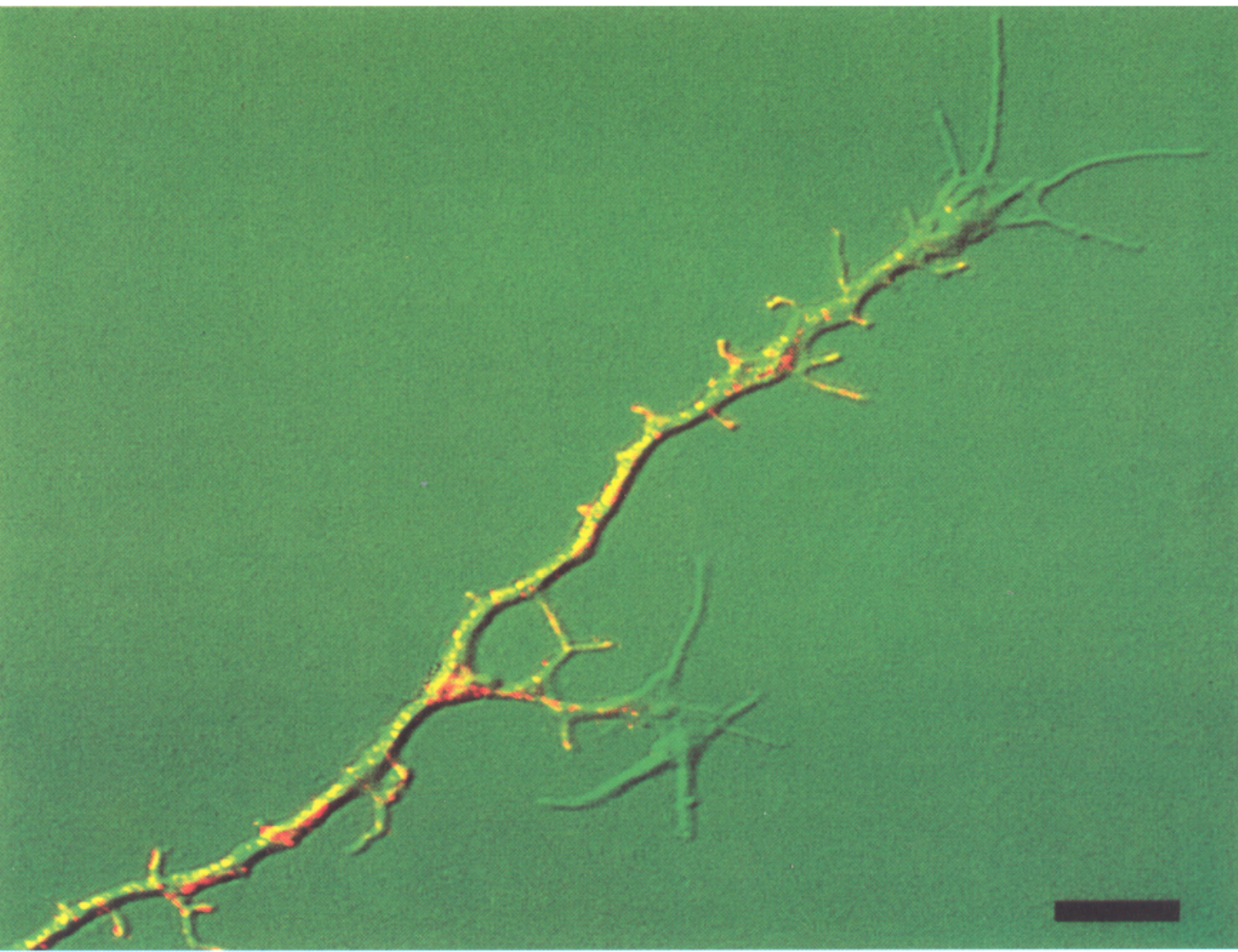



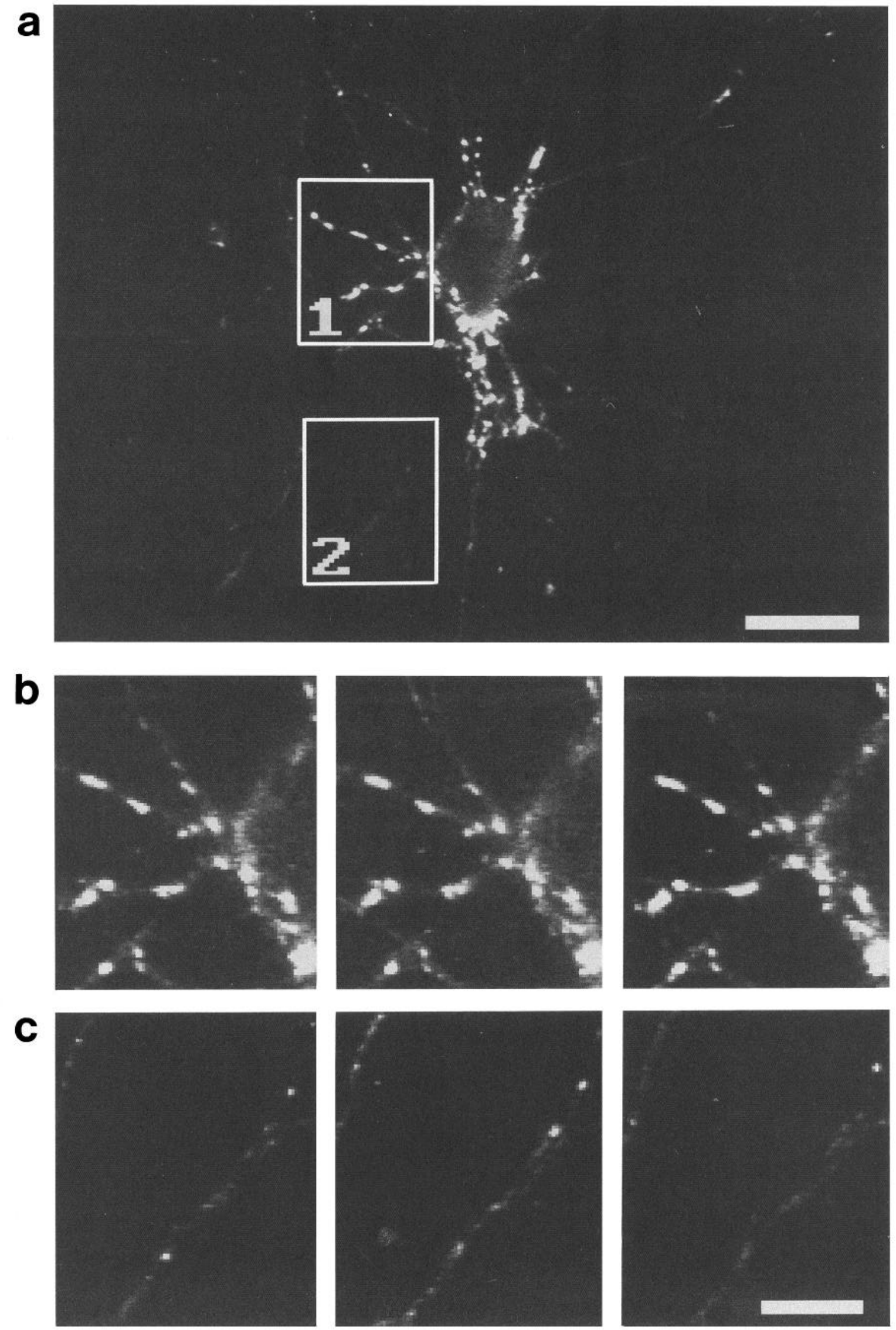

Figure 3. Motility of structures labeled by CY3-Syt-Abs in mature neurons. $a$, Low power field of a neuron ( 8 DIV) which receive multiple synaptic contacts imaged with an SIT camera. The culture was preincubated for 20 min with the antibodies in KRH-high $\mathrm{K}^{+}$. Large bright dots of 

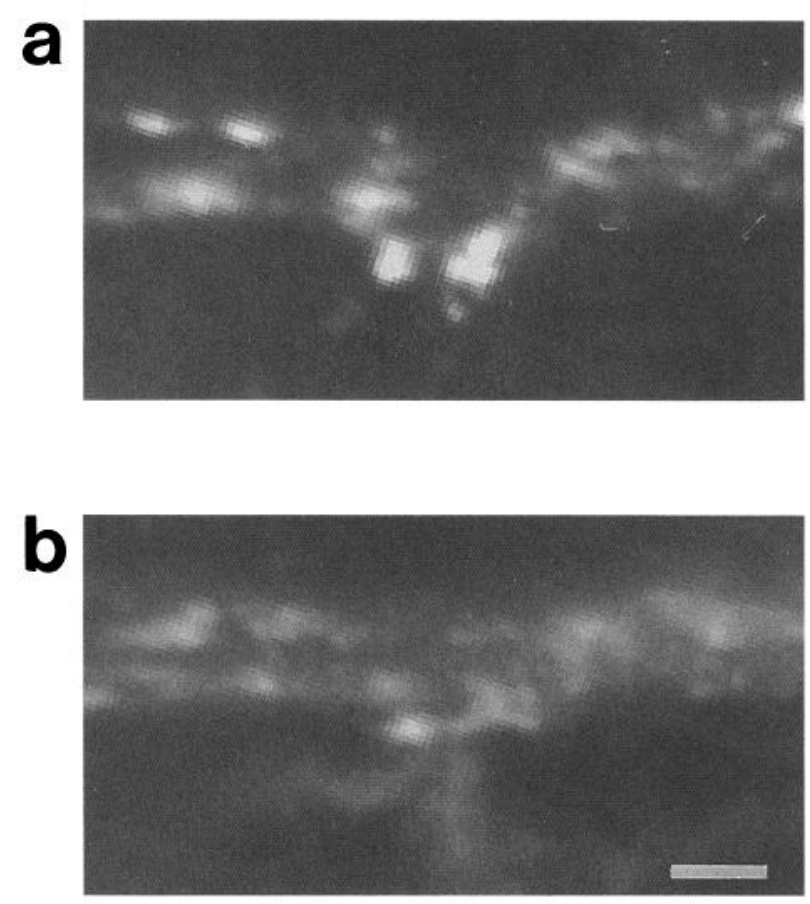

Figure 4. Effect of okadaic acid on presynaptic SV clusters labeled by CY3-Syt $\mathrm{C}_{1}$-Abs $\left(20 \mathrm{~m}\right.$ in $\mathrm{KRH}$-high $\left.\mathrm{K}^{+}\right)$in $8 \mathrm{DIV}$ neurons. $a$, High magnification of a segment of a dendrites which receives a few synaptic inputs. $b$, Same field as $a 20 \mathrm{~min}$ after application of okadaic acid (2 $\mu \mathrm{M})$.

of Figure $3 a$ taken at 2 min intervals are shown in Figure 3, $b$ and $c$. No change in the position of the large spots is visible (Fig. $3 b$ ) and we have not seen any displacement of these structures greater than $1 \mu \mathrm{m}$ during a $1 \mathrm{hr}$ recording. This observation is consistent with the notion that large fluorescent dots represent presynaptic clusters of SVs. In contrast, the smaller fluorescent dots present in isolated segments of axons exhibited a high degree of motility both in anterograde and retrograde directions with frequent switches in direction. A significant change in the relative positions of these spots is clearly visible in Figure $3 c$. Since axons are very thin and lie directly at the surface of the coverslip, the observed displacement cannot be explained by small changes in the focal plane during the observation interval. At continuous recording, particle movement was found to be smooth but was frequently interrupted by stops and by reversions in the direction of movement. The maximal speed of the smooth displacements was in the range of $1 \mu \mathrm{m} / \mathrm{sec}$ consistent with microtubule-based motility (Vale, 1987).

It was previously reported that presynaptic clusters of synaptic vesicles at the frog neuromuscular junction can be disrupted by exposure to okadaic acid (Betz and Henkel, 1994), a compound which is thought to act by inhibiting protein phosphatases (Bialojan and Takai, 1988). In these nerve terminals application of $2 \mu \mathrm{M}$ okadaic acid induced a disorganization of the clusters and a nonrandom redistribution of the vesicles into large clusters as if they "were swept along by an active translocation mecha- nism" (Betz and Henkel, 1994). Okadaic acid had a similar effect when applied to hippocampal neuronal cultures (8 DIV neurons). The large dots of CY3-Syt,-Abs accumulation were disrupted after $20 \mathrm{~min}$ exposure to $2 \mu \mathrm{M}$ okadaic acid and a different nonrandom distribution of the dye was visible after this treatment (Fig. 4). These observations demonstrate a similar effect of okadaic acid at peripheral (Betz and Henkel, 1994) and central synapses and further corroborate the conclusion that large dots of $\mathrm{CY} 3$ labeling represent presynaptic synaptic vesicle clusters.

\section{Quantitative measurement of CY3-Syt $t_{-}$Ab uptake at synapses}

We next investigated whether uptake of CY3-Syt-Abs at presynaptic spots could be used to quantify synaptic vesicle exocytosis. To this aim we developed a double immunofluorescence procedure by which CY3-Syt 1 -Abs uptake could be normalized to the total synaptotagmin I content of a given nerve terminal. This content is variable due to the heterogeneous level of synaptotagmin I expression in neurons and to variations in size of presynaptic SV clusters (Matteoli et al., 1992). Cultures were incubated with CY3-Syt $-\mathrm{Abs}$ for various time intervals and in media with different ionic compositions. Then, they were fixed, permeabilized, and counterstained with a monoclonal antibody directed against the cytoplasmic domain of the protein (Syt ${ }_{\mathrm{c}}$ Abs) followed by fluorescein-conjugated secondary antibodies. The fluorescein signal thus obtained provided a value to normalize levels of CY3 uptake. The ratio between the CY3 and the fluorescein signals measured on individual nerve terminals with a CCD camera is expected to closely reflect the level of SV exocytosis.

Figure 5 shows an example of the images used for the quantification. Left panels represent images from four different neuronal cultures ( 8 DIV, stage 5 neurons) incubated with CY3Syt $-\mathrm{Abs}$ for $20 \mathrm{~min}$ in different experimental conditions. The right panels are corresponding fluorescein images revealing the total amount of Synaptotagmin in a nerve terminal. The uptake of CY3-Syt $-\mathrm{Abs}$ is clearly higher in $\mathrm{KRH}$ high $\mathrm{K}^{+}$(Fig. $5 c$ ) than in KRH (Fig. 5a). An even lower level of CY3-Syt - Abs internalization was observed when cells were incubated in KRHzero $\mathrm{Ca}^{2+}$ (Fig. $5 e$ ) and only a very low level of fluorescence was present on cells incubated with the CY3-Syt $-\mathrm{Abs}$ at $4^{\circ} \mathrm{C}$ (Fig. $5 g$ ).

The ratios for these different treatments after incubation with CY3-Syt-Abs for 5, 20, and $60 \mathrm{~min}$ are shown in Figure $6 a$. At $5 \mathrm{~min}$, the ratios observed in control $\mathrm{KRH}$ and $\mathrm{KRH}$-zero $\mathrm{Ca}^{2+}$ were only slightly higher than the ratios observed on randomly selected portions of neurons incubated with $\mathrm{KRH}$ at $4^{\circ} \mathrm{C}$, that is, conditions in which no exocytosis takes place and in which, accordingly, no fluorescent "hot spots" were detectable. In contrast, a much higher ratio was measured in cultures incubated in depolarizing conditions in $\mathrm{KRH}-\mathrm{high} \mathrm{K}^{+}$. However when $\mathrm{KRH}-$ high $\mathrm{K}^{+}$was applied in the absence of $\mathrm{Ca}^{2+}$ (zero $\mathrm{Ca}^{2+}, 1 \mathrm{mM}$ EGTA) the rate of exocytosis was the same as in $\mathrm{KRH}-\mathrm{zero} \mathrm{Ca}^{2+}$ (not shown). At longer incubation times, the ratios in KRH-high $\mathrm{K}^{+}$continued to increase, but at a lower rate. A steady increase in the ratios was also observed in cultures incubated with control

fluorescence visible on the perikaryon and in the dendrites (e.g., rectangle 1 ) represent SV clusters in presynaptic nerve terminals. Smaller, less intensely fluorescent dots visible on axons (e.g., rectangle 2) probably represent endosomes or smaller SV clusters. $b$ and $c$, High magnification views of the two areas enclosed by rectangles in $a$ shown at three different 2 min intervals. Presynaptic clusters are stationary $(b)$, while smaller dots visible in axons exhibit high motility $(c)$. Scale bars: $a, 20 \mu \mathrm{m} ; b$ and $c, 10 \mu \mathrm{m}$. 

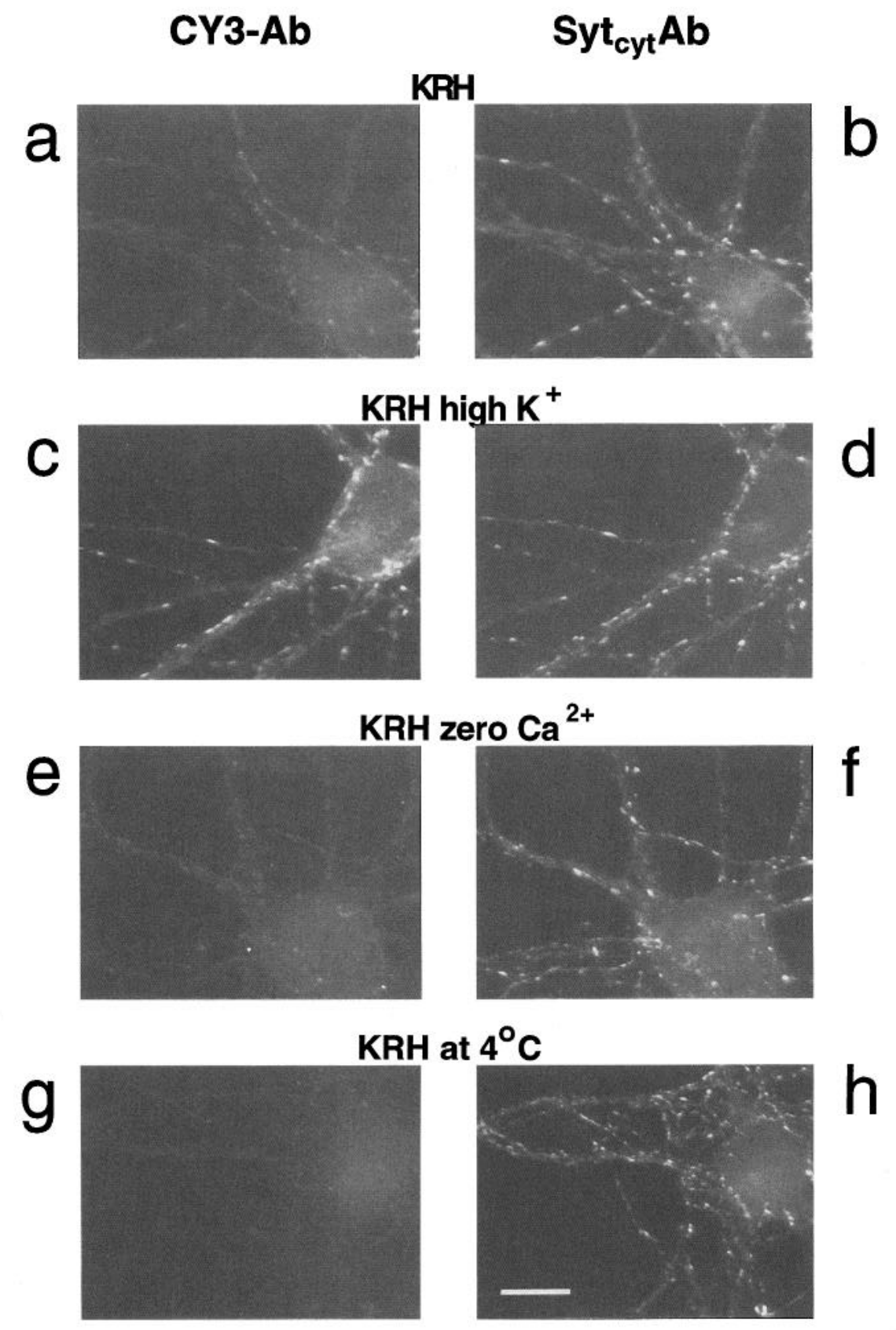

Figure 5. Synaptic labeling by CY3Syt - -Abs is activity dependent. Fluorescent images taken by a CCD camera from cultures rich in synaptic contacts (8 DIV) incubated for $20 \mathrm{~min}$ with CY3-Syt,-Abs in KRH, $a$ and $b$; KRH high $\mathrm{K}^{+}, c$ and $d$; $\mathrm{KRH}$-zero $\mathrm{Ca}^{2+} e$ and $f ; \mathrm{KRH}$ at $4^{\circ} \mathrm{C}, g$ and $h$. At the end of the incubation cells were fixed, permeabilized and counterstained (fluorescein) with $\mathrm{Syt}_{c}-\mathrm{Ab}$. Left images represent $\mathrm{CY} 3$ signal and right images fluorescein signal. Scale bar, $10 \mu \mathrm{m}$.
$\mathrm{KRH}$ and $\mathrm{KRH}$-zero $\mathrm{Ca}^{2+}$, but in this case the sharp increase during the first $5 \mathrm{~min}$ was not present. This finding indicates that the major stimulatory effect of high $\mathrm{K}^{+}$is of short duration, as expected by the known property of $\mathrm{Ca}^{2+}$-evoked neurotransmitter release to undergo rapid inactivation (Liley, 1956; Zuker et al., 1991). At longer times the ratio measured in $\mathrm{KRH}$-zero $\mathrm{Ca}^{2+}$ tended to be lower than the ratio observed in control KRH (although it was statistically different only at $20 \mathrm{~min}$ ), possibly reflecting the occurrence of spontaneous synaptic activity in the cultures. The depolarization-dependent increase in CY3-Syt $-\mathrm{Ab}$ uptake was completely blocked by a $1 \mathrm{hr}$ pretreatment of the cultures with tetanus toxin, Botulinum D, and F toxins (Fig. 7, and not shown).

The observation that $\mathrm{CY} 3-\mathrm{Syt}_{1}-\mathrm{Ab}$ uptake in $\mathrm{KRH}$-high $\mathrm{K}^{+}$ continues to increase during $60 \mathrm{~min}$ incubation contrasts with the time course of the labeling and unlabeling with the styryl dye FM1-43 of presynaptic clusters of hippocampal neurons in strongly depolarizing conditions (Ryan et al., 1993). Experiments carried out with this dye have suggested that the bulk of the SV population present in a nerve terminal undergoes exocytosis within less than $1 \mathrm{~min}$ of an incubation in high $\mathrm{K}^{+}$(Ryan et al., 1993). We have confirmed these findings in our cultures. In the same $\mathrm{KRH}$-high $\mathrm{K}^{+}$medium used for the labeling with CY3-Syt - Abs, we have observed a saturation of presynaptic labeling by FM1-43 after less than 2 min (not shown). To confirm that FM1-43 and CY3-Syt - Abs stain the same structures, we performed double labeling experiments. Neurons first labeled in vivo with CY3-Syt - Abs were subsequently labeled with FM143. As shown in Figure 8, a substantial colocalization was observed between hot spots of FM1-43 and large dots positive for CY3. Large FM1-43 positive spots, which are negative for CY3Syt $\mathrm{t}_{1}$-Abs internalization, most likely correspond to nerve terminals of neurons which do not express significant levels of synaptotagmin I. Most likely, the different labeling kinetics reflect 


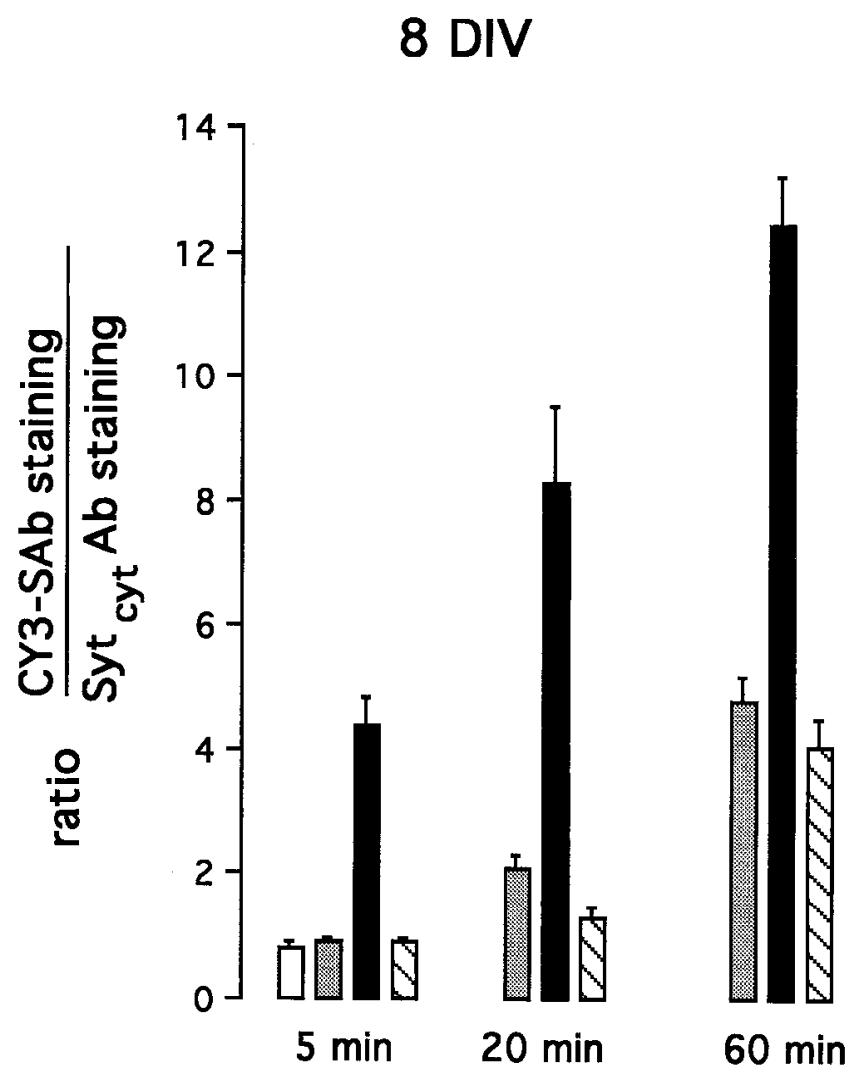

Figure 6. Quantitative analysis of CY3-Syt - Abs labeling at synapses of mature ( $8 \mathrm{DIV}$ ) neurons. Cultures were incubated for the times indicated with $\mathrm{CY} 3-\mathrm{Syt}_{1}-\mathrm{Abs}$ in $\mathrm{KRH}, \mathrm{KRH}$-high $\mathrm{K}^{+}, \mathrm{KRH}-z e r o \mathrm{Ca}^{2+}$, and $\mathrm{KRH}$ at $4^{\circ} \mathrm{C}$. After a brief wash cells were then fixed, permeabilized and counterstained with $\mathrm{Syt}_{\mathrm{c}}-\mathrm{Ab}$ (fluorescein). Images were collected with a CCD camera. Bars represent ratios between the CY3 and the fluorescein fluorescence. Error bars represent SE.

the very different nature of the two labeling compounds (see Discussion).

In vivo labeling of $S V s$ in isolated neurons: presence of motile SV clusters

We next investigated the dynamics of synaptic vesicle labeling by CY3-Syt - Abs in axons of neurons developing in isolation. CY3-Syt 1 -Abs were applied to the medium of stage 3 neurons (3 DIV) according to the classification of Dotti et al. (1988). At this stage cells are already well polarized and have both axons and dendrites, but synaptic contacts have not yet formed and many axons are still completely isolated. As previously shown, CY3-Syt - -Abs are taken up efficiently by these axons and primarily by their distal portions (Fig. 9; see also Matteoli et al., 1992). Figure $2 b$ shows the distal segment of an axon from a culture which has been incubated with CY3-Syt - Abs and im aged with a CCD camera as described for the neuron shown in Figure $2 a$. The DIC image shows the presence of two growth cones with typical distal navigating filopodia plus several shorter filopodia emerging from the axon shaft. The fluorescent image

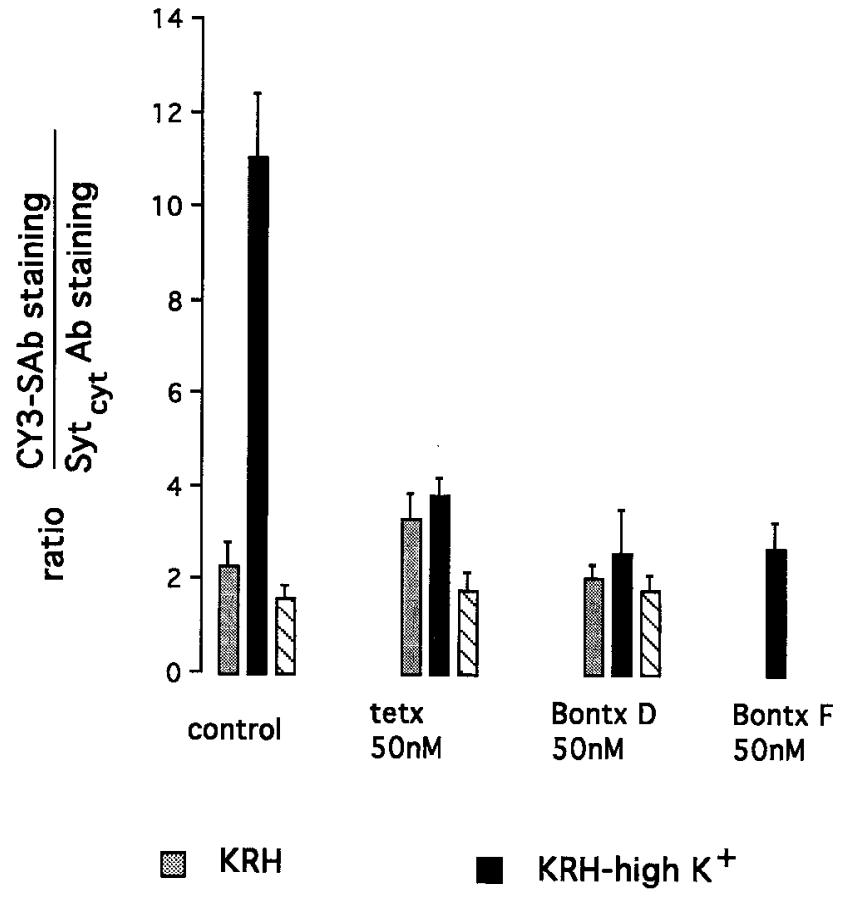

Figure 7. Effect of clostridial neurotoxins on the uptake of CY3-Syt Abs at synapses; 9 DIV neurons were pretreated with toxins for $1 \mathrm{hr}$ and then incubated for 20 min with CY3-Syt $-\mathrm{Abs}$ in the media indicated. Quantitative analysis was performed as indicated in the legend of Figure 6.

reveals the presence of an heterogeneous population of CY3labeled structures ranging from very fine puncta to larger dots. Many of the fluorescent particles are present in the short filopodia emerging from the axon shaft. These filopodia were typically stable in shape for minutes and were clearly anchored to the substrate. Fluorescent particles were excluded, however, from the leading edge of the growth cone of the axon (a region about $10 \mu \mathrm{m}$ in length) and from the highly dynamic filopodia emerging from the axon tip which appear to guide the axon in its navigation (Fig. 2b). These filopodia were not anchored to the glass and underwent frequent lateral displacements. This observation is consistent with the finding that filopodia of growth cones exclude membranous organelles (Forscher and Smith, 1988; Mundigl et al., 1993).

All fluorescent particles visible in the distal segments of the axons were highly motile, as shown by six time lapse images (taken at 2 min interval) of the field enclosed by a rectangle in Figure $9 a$. In the axonal shaft, fluorescent particles underwent discontinuous movements along the major axis of the axon, both in the anterograde and the retrograde direction with frequent changes in the direction of movement. The result was a continuous intermixing of particles within a section of the distal axon, with no obvious net movement of particles in one direction. Movement of fluorescent particles was also visible in the short filopodia (Fig. 9b), where particles underwent saltatory movement from the tip to the base of the process. Since these filopodia are static and anchored to the coverslips, the translocation of fluorescent particles along them cannot be explained by filopodia displacement but must reflect movement inside the filopodia. These short filopodia contained at least one microtubule as demonstrated by counterstaining with anti-tubulin antibodies 

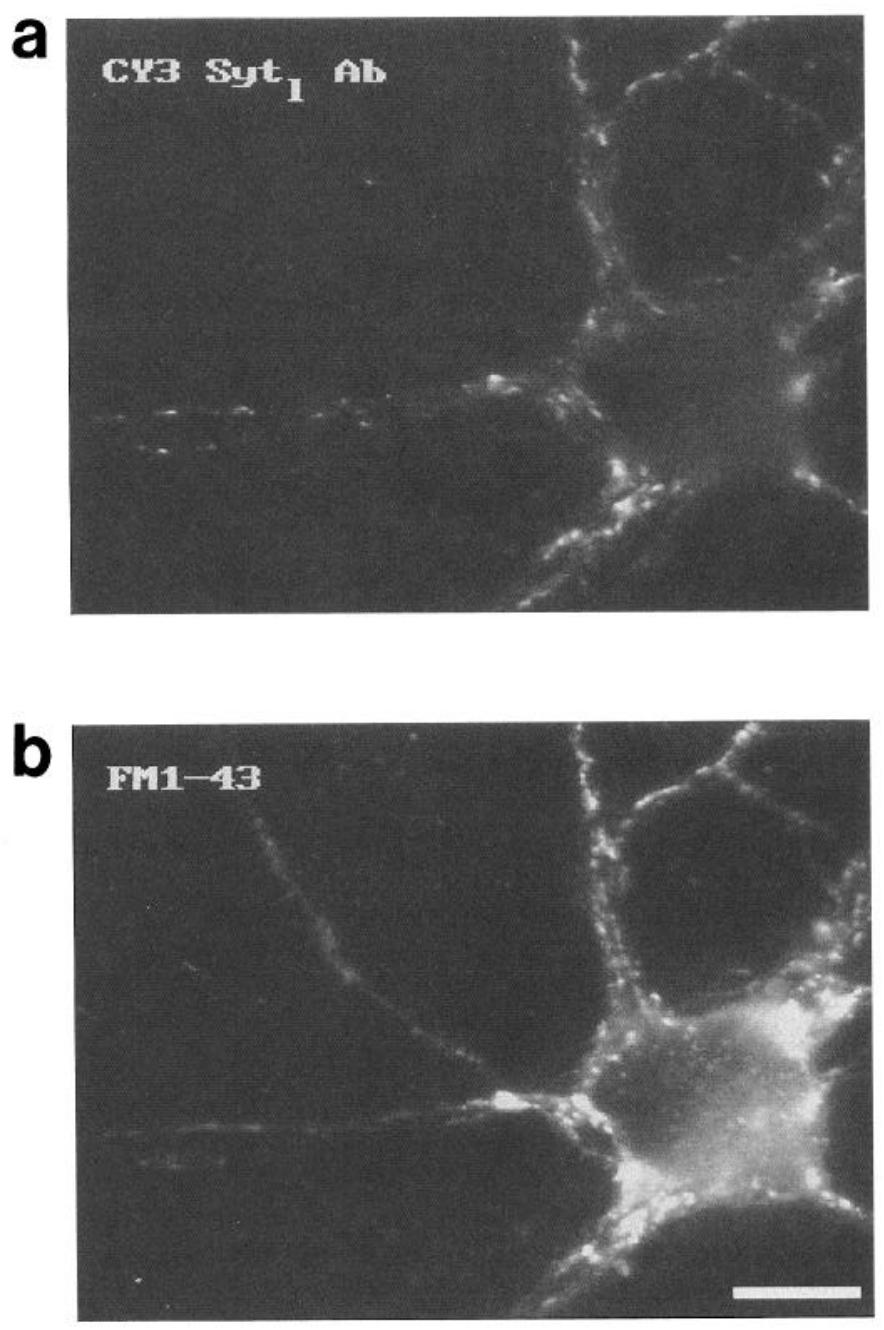

Figure 8. Comparison of the fluorescent signal produced by the uptake of CY3-Syt-Abs and of FM1-43 in 8 DIV neurons. The culture was first incubated for $20 \mathrm{~min}$ in $\mathrm{KRH}$-high $\mathrm{K}^{+}$containing CY3-Syt $\mathrm{C}_{1}-\mathrm{Abs}$. After collecting the image shown in $a$ with a CCD camera, the culture was incubated with FM1-43 for 2 min in $\mathrm{KRH}$-high $\mathrm{K}^{+}$, washed in $\mathrm{KRH}$, and imaged with a CCD camera for the FM1-43 signal $(b)$. Scale bar, $2 \mu \mathrm{m}$.

after fixation (not shown; see also the EM micrograph of Fig. $10 a$ ). This finding is consistent with the possibility that even particle movement observed in filopodia may be accounted for by microtubule-based motors. Particles present both in the axonal shaft and in filopodia often split into smaller particles which then underwent independent movement. Conversely, small particles which coalesced into large particles were seen.

Counterstaining of CY3-Syt 1 -Abs labeled neurons for other SV proteins (e.g., synaptophysin, SV2; Matteoli et al., 1992) after fixation and permeabilization, demonstrated a precise colocalization indicating that all CY3-labeled structures coincide with SV membranes (not shown). Individual spots, however, were too bright to be represented by single SVs. To determine whether spots visible by light microscopy could be represented by clusters of SVs which move in bulk, we analyzed a large number of distal axonal segments by EM. In all cases, clusters of SVs, were visible both in the shaft and in filopodia (Fig. 10), although not in the filopodia of the growth cone (Mundigl et al., 1993). When cultures had been incubated for $1 \mathrm{hr}$ prior to fix- ation with the fluid phase marker horseradish peroxidase (HRP), a significant number of vesicles was labeled by HRP reaction product (Fig. 10b,c,i,j), indicating that they undergo exo-endocytosis (Ceccarelli et al., 1973; Heuser and Reese, 1973). In some experiments, neurons were processed by a procedure which results in the specific labeling by gold particles of SVs which have undergone at least two complete rounds of recycling (Matteoli et al., 1992): neurons were first incubated with Syt - $^{-}$ Abs (1 hr) to load SVs with IgG, then briefly washed, incubated with protein A gold particles (1 hr) to label antibody-containing vesicles which become reexposed by exocytosis, and finally fixed. After such treatment, many vesicles contained gold particles (Fig. 10d-f), confirming their identification as SVs. These findings strongly suggest that a large fraction of the motile fluorescent puncta visible in axon shafts and filopodia represent SV clusters that behave as a coherent unit and are not stably anchored to a given region of the cell surface. These clusters, however, can dynamically fragment into smaller units or coalesce into larger ones.

To confirm these interpretations and to determine further whether SV clusters in isolated axons had similar properties to presynaptic SV clusters, we examined the effect of okadaic acid on these motile puncta. As shown in Figure 11, a $20 \mathrm{~min}$ exposure to $20 \mu \mathrm{M}$ okadaic acid induced a dimming of the bright spots as one would expect from a dispersal of SVs. These changes preceded drastic structural changes induced by okadaic acid in distal axons such as growth cone collapse and eventually axon retraction (not shown).

\section{CY3-Syt - A b uptake by isolated axons is stimulated by depolarization}

We then investigated whether uptake of CY3-Syt $-\mathrm{Abs}$ by developing neurons was depolarization and $\mathrm{Ca}^{2+}$-dependent. Stage 3 neurons ( 3 DIV) were incubated in the same conditions described above for stage 5 neurons. Ratios between the CY3 and the fluorescein signals obtained by the CCD camera on randomly selected fluorescent "hot spots" in distal axonal segments were calculated and are shown in Figure 12. As in the case of synaptic contacts of stage 5 neurons, a depolarizing medium (KRH-high $\mathrm{K}^{+}$) produced a higher ratio than $\mathrm{KRH}$ or $\mathrm{KRH}-$ zero $\mathrm{Ca}^{2+}$ and this effect was not seen using $\mathrm{KRH}$-high $\mathrm{K}^{+}$with zero $\mathrm{Ca}^{2+}$. In all conditions the ratios increased in parallel with times of incubation (Fig. 12). The main difference between results obtained in isolated axons and those obtained at mature synapses, is that ratios at $5 \mathrm{~min}$ in control $\mathrm{KRH}$ were already significantly higher than the control ratios on randomly selected neuronal regions at $4^{\circ} \mathrm{C}$, indicating a higher level of constitutive SV exocytosis. Similar results were obtained in $\mathrm{KRH}-z e r o \mathrm{Ca}^{2+}$.

\section{Quantification of elementary fluorescence units at synapses and in "hot spots" of isolated axons}

The findings reported above do not provide any information on the number of antibody molecules responsible for vesicle labeling. The evaluation of this number could help to understand in molecular terms the process of vesicle labeling by CY3-Syt Abs. We attempted, therefore, to determine the elementary fluorescence unit of CY3-Syt - Abs. A unitary element of fluorescence was indeed observed and measured by analyzing with the CCD camera the background fluorescence produced by CY3Syt $_{1}$-Abs on glass coverslips (see Materials and Methods and Fig. 1). The value of such unit was independent of antibody concentration and time of incubation of the antibody with the 
coverslip, but varied using conjugates with different CY3/IgG stoichiometric ratio (not shown and Fig. 1). This value appears therefore to represent a single IgG conjugate. Although this conclusion deserves further investigation, such a value allowed us to obtain a minimal estimate of the number of conjugates present in fluorescent particles of neurons.

Measurements of fluorescence intensity, expressed as numbers of elementary units, in old (stage 5: 8 DIV) and young (stage 3: 3 DIV) neurons which had been incubated with CY3-SytAbs for $20 \mathrm{~min}$ in $\mathrm{KRH}$-high $\mathrm{K}^{+}$are shown in Figure 13. In 8 DIV neurons, number of units on synaptic clusters is in the hundreds range (200-250), while values on motile particles in nonsynaptic regions are about 15-20 times lower (Fig. 13a). It has been estimated that the number of SVs in presynaptic terminal of the CNS is in the range of 200 (Harris and Stevens, 1989). Thus, after 20 min of exposure to the antibodies in depolarizing media, the number of fuorescence units per average nerve terminal is in the same range of the number of SVs. In control experiments CY3-Syt t $_{1}$ Abs were applied to the same cultures only after formaldehyde fixation and Triton X-100 permeabilization. Under these conditions, the average maximal number of fluorescence units of CY3-Syt,-Abs which could be bound to presynaptic nerve terminals was about 1500 . Considering that even this value must represent a great underestimation of the total number of binding sites for CY3-Syt $\mathrm{C}_{1}$-Abs present in nerve terminals due to steric hindrance resulting from fixation (De Camilli et al., 1983), it is conceivable that the fluorescence unit measured by us may indeed represent a single IgG conjugate. In experiments in which neurons were first incubated for $1 \mathrm{hr}$ with $\mathrm{Syt}_{1}$-Abs, and then for an additional hr with protein A-gold conjugates, few gold particles per vesicles were observed (see above). Thus, although electron microscopy and fluorescence data cannot be directly compared, the results of the two techniques are in good agreement.

Fluorescent intensity of "hot spots" visible in filopodia of three DIV neurons was about 10-15 times lower than fluorescence levels on presynaptic clusters, in agreement with the smaller number of SVs forming these aggregates (Fig. 13b).

\section{Discussion}

In the present study we have validated the use of CY3-Syt - Abs as a "vital" dye for SVs which can be used to quantify SV exocytosis and to study the traffic of SVs in the living cell. We demonstrated that this probe is very effective in monitoring long lasting changes in the rate of SV exocytosis. Additionally, new information obtained with this probe concerning the dynamics of SVs in isolated axons of developing neurons is reported.

Application of CY3-Syt - Abs to cultures after formation of synaptic contacts resultcd in a hcavy labcling at sites of contacts between axons and dendrites. Large immobile fluorescent structures visible at these sites were also positive for other SV markers and correspond therefore to SV-filled nerve terminals as seen by electron microscopy (Fletcher et al., 1991). They also overlapped with the bright fluorescence puncta which become labeled by FM1-43, although the latter dye labeled a greater number of synapses, a finding explained by the known expression of synaptotagmin I only in a subpopulation of nerve terminals (Wendland et al., 1991; Matteoli et al., 1992). Labeling of the presynaptic structures was depolarization and $\mathrm{Ca}^{2+}$ dependent and could be inhibited by pretreatment of neurons with clostridial neurotoxins, which are potent inhibitors of neurotransmitter release (Niemann, 1991; Montecucco and Schiavo, 1993).
The time course of labeling with CY3-Syt $-\mathrm{Abs}$ was quite different from the time course of the labeling with FM1-43 of CNS synapses in culture (Ryan et al., 1993). In KRH-high $\mathrm{K}^{+}$, labeling with FM1-43 of an average nerve terminal is virtually saturated within less than 1 min (Betz et al., 1992a; Betz and Bewick, 1993; Ryan et al., 1993; our unpublished observations). Likewise, unloading of the bulk of the dye from a prelabeled nerve terminal in KRH-high $\mathrm{K}^{+}$occurs in the same time range. These findings imply a nearly complete turnover of the SV content of a terminal in less than one minute (Ryan et al., 1993).

While uptake of CY3-Syt - Abs was strongly stimulated in the first few minutes after application of $\mathrm{KRH}^{-h i g h ~ \mathrm{~K}^{+}}$, the level of uptake was clearly far from saturation. The level of CY3 fluorescence on each terminal steadily increased, although at a lower rate, for at least $60 \mathrm{~min}$ after the initial burst following the application of a depolarizing medium. This lower rate was still higher than the rate of uptake seen in preparations incubated in control KRH, which must reflect basal exocytosis. These findings may be explained by relatively slow kinetic of antibody binding compared to the rapid "on" and "off" rate of FM1-43 and by the very transient exposure at the cell surface of SV membranes (Torri-Tarelli et al., 1990). While the short time that the SV membranes spend at the cell surface may be sufficient to allow nearly complete binding of FM1-43, this time might be far too short for a saturation of synaptotagmin molecules with CY3-Syt $t_{1}$-Abs. In fact, after $20 \mathrm{~min}$ in $\mathrm{KRH}$-high $\mathrm{K}^{+}$, the average number of elementary fluorescence units within a nerve terminal was about 250 . This number is far below the average number of fluorescence units which can be bound by nerve terminals after fixation and permeabilization and is in the range of SVs present in a varicosity (Harris and Stevens, 1989).

In principle one could explain the different time-course of nerve teminal labeling by CY3-Syt - Abs and by FM1-43 with the hypothesis that most exocytotic events simply involve a fusion pore which is not permeable to large macromolecules (Fesce et al., 1994). However, the different nature of the two dyes is sufficient to explain the difference in the time course of uptake without postulating the occurrence of two types of exocytotic events. FM1-43 is clearly more suited to detect changes in the rate of SV exocytosis which occur with fast kinetics. CY3-Syt Abs are an useful tool to monitor long term changes in synaptic activity. In our study they revealed a persistent increase in the rate of SV exocytosis during prolonged exposure to depolarization. These changes could not be recorded by the FM1-43 signal which is rapidly saturated.

While the properties of FM1-43 offer advantages for studies on the kinetic parameters of neurotransmitter release at synapses, the nonspecific binding of this dye to all membranes limits its use for studies at sites where it is not clear whether SVs represent the predominant exo-endocytotic traffic. CY3-Syt $-\mathrm{Abs}$, in contrast, are highly specific marker for SV membranes and can be used to study their exo-endocytosis irrespective of the concomitant occurrence of exo-endocytosis of other vesicular carriers. Furthermore, CY3-Syt $-\mathrm{Abs}$, which remain associated with SVs during repeated cycles of exo-endocytosis, are an optimal tool to monitor specifically the traffic of SVs over long incubation times. In this study we have used CY3-Syt $\mathrm{C}_{1}-\mathrm{Abs}_{\text {to }}$ obtain new insights into the properties of SVs in developing isolated axons before synapse formation.

First, the accumulation of CY3-Syt - Abs at axonal "hot spots" which are highly motile within the axons revealed the property of these organelles to cluster with each other even in 

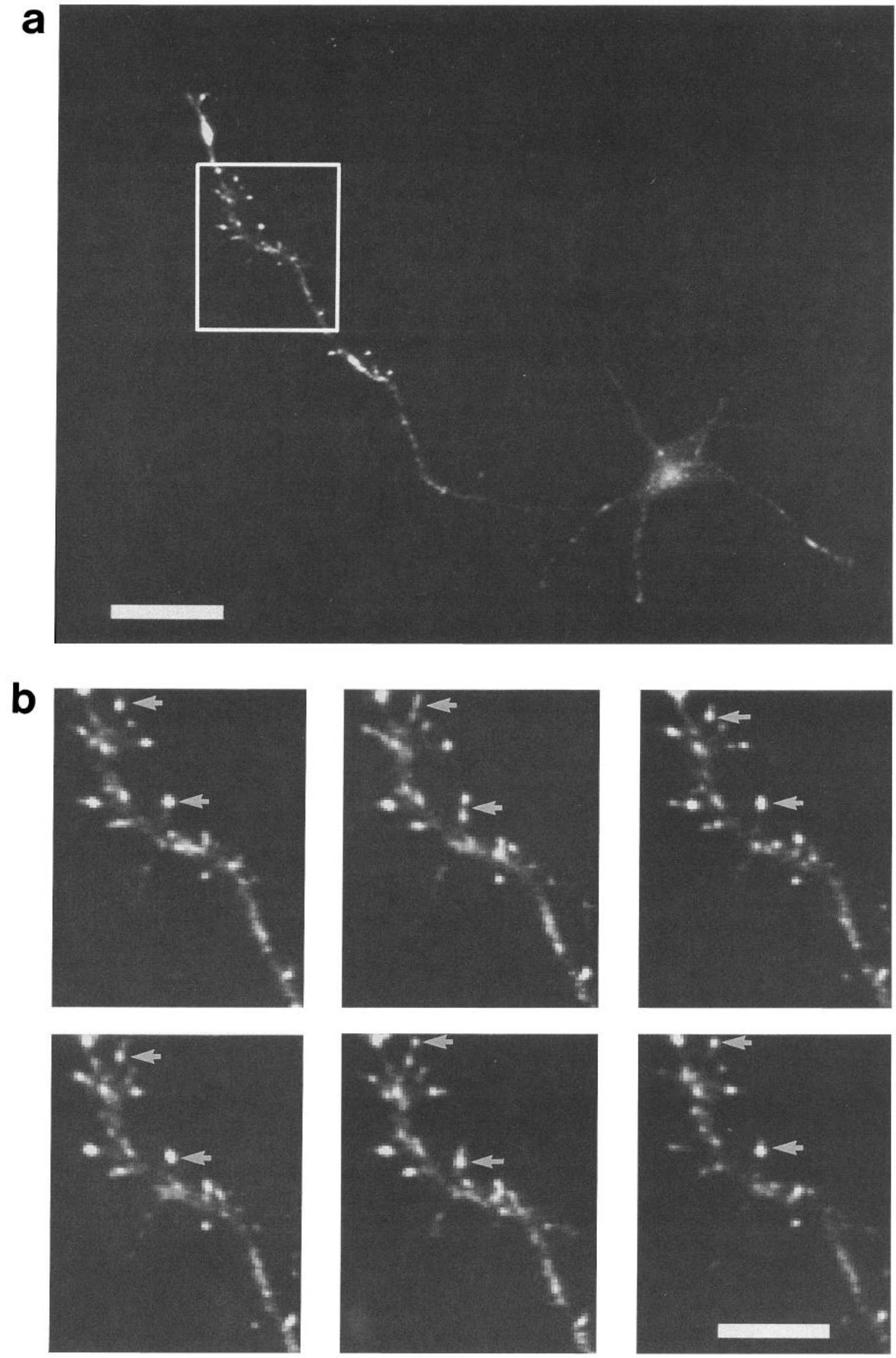

Figure 9. Motility of structures labeled by CY3-Syt $t_{1}$-Abs in the axon of an isolated neuron ( 3 DIV). The culture was incubated with labeled antibodies for $20 \mathrm{~min}$ in $\mathrm{KRH}$-high $\mathrm{K}^{+}$prior to imaging with an SIT camera. A low power view of the neuron is shown in $a$. The largest and more 

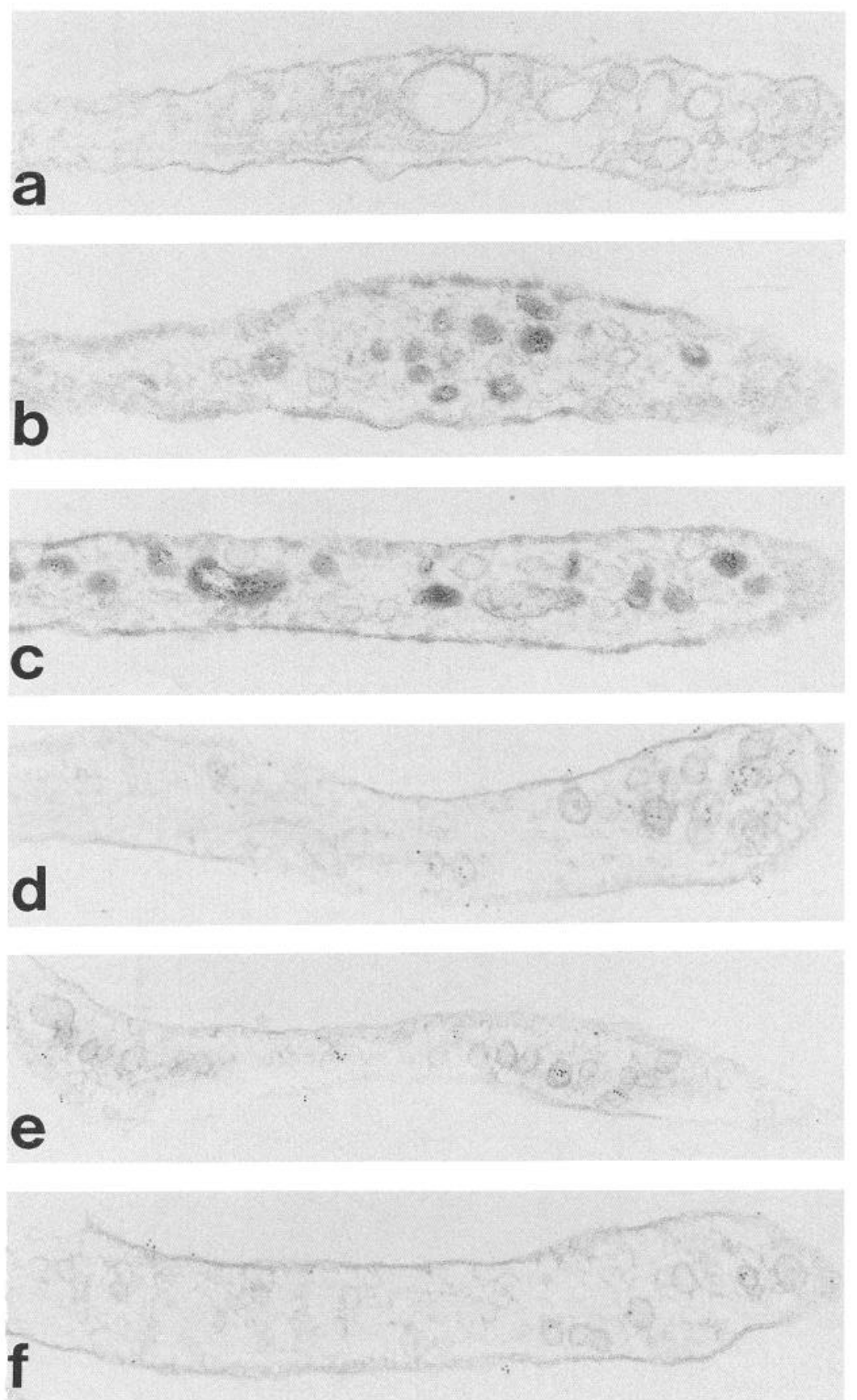
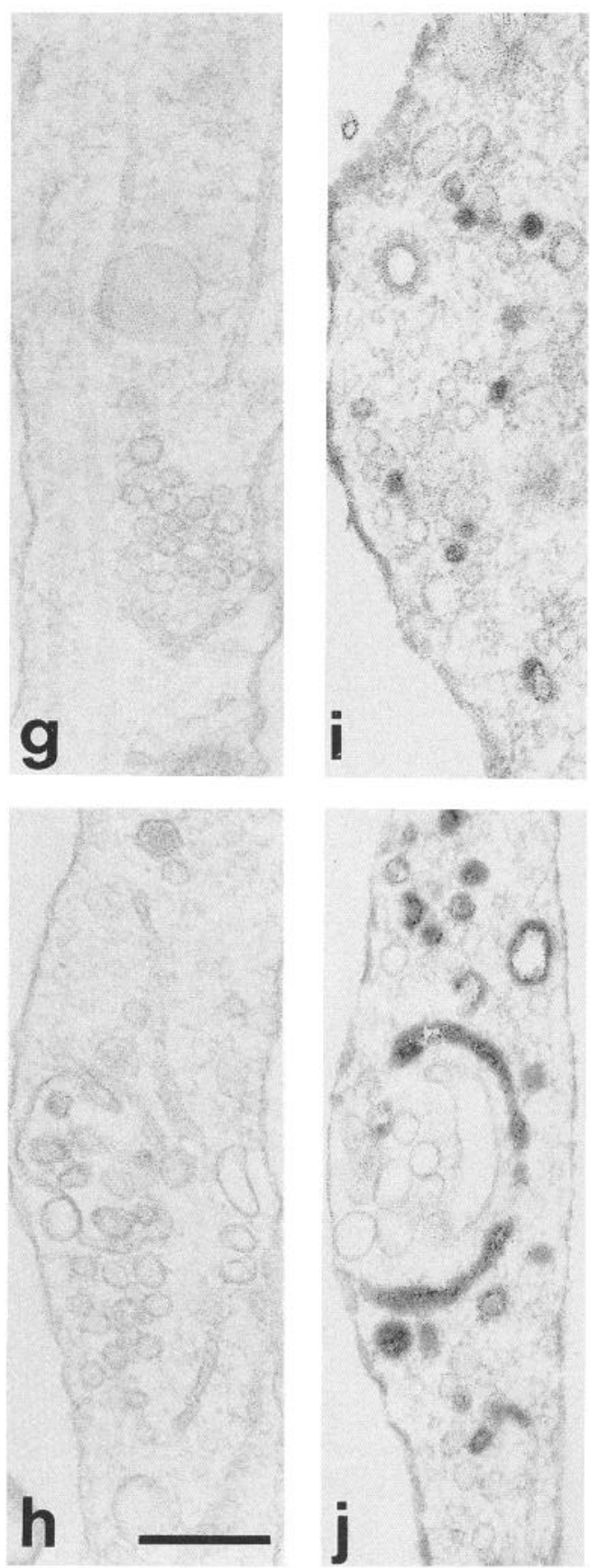

Figure 10. Gallery of EM micrographs illustrating the presence of SV clusters in distal segments of isolated axons and in their filopodia. Filopodia are shown in $a-f$ and axonal shafts in $g-j . a, g$, and $h$, The neuronal growth medium was directly substituted with fixative. $b, c, i$, and $j$ are from cultures were incubated with horseradish peroxidase (HRP) and KRH-high $\mathrm{K}^{+}$for $1 \mathrm{hr}$ prior to fixation. $d-f$, Neurons were incubated with Syt, Abs (1 hr), then briefly washed, incubated with protein A-gold particles (1 hr), and fixed. Clusters of SVs are visible both in the axonal shaft and in filopodia. In $c$ and $j$ endosome-like structure surrounded by SVs are visible. A multivesicular body is shown in $h$. Note presence of a microtubule in $a$. Scale bar, $200 \mathrm{~nm}$.

the absence of synaptic contacts. The existence of these clusters was confirmed by electron microscopy. Thus, formation of the clusters is not driven by a contact with a postsynaptic membrane. This finding has implications for the elucidation of the mecha- nism of assembly and biochemical nature of the matrix which connects the vesicles with each other. It suggests that the formation of the large presynaptic clusters may result from the coalescence of packages of SVs rather than from the recruitment

$\leftarrow$

intensely fluorescent CY3-labeled structures are concentrated in the distal axon and in its filopodia. $b$, High magnification of six time-lapse images taken at $2 \mathrm{~min}$ intervals of the field enclosed by a rectangle in a. Fluorescent particles exhibit extensive motility. Arrows point to two particles which display substantial changes during the observation. Scale bars: $a, 20 \mu \mathrm{m} ; b, 10 \mu \mathrm{m}$. 

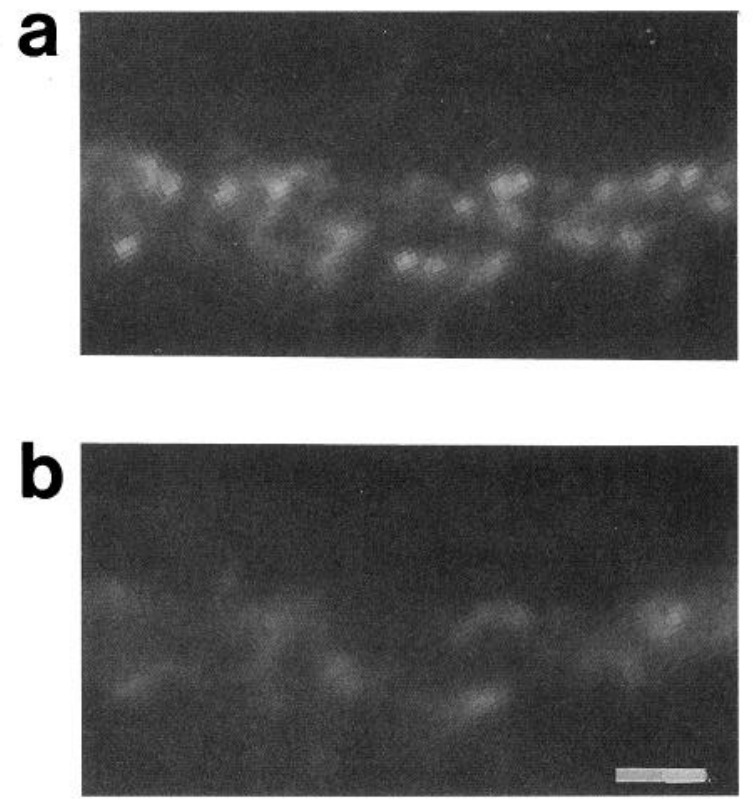

Figure 11. Effect of okadaic acid on bright spots of fluorescence visible in the axons of an isolated neuron. A 3 DIV culture was incubated with CY3-Syt $-\mathrm{Abs}$ for $20 \mathrm{~min}$ in $\mathrm{KRH}^{-}$high $\mathrm{K}^{+} . a$, High magnification of a distal segment of an isolated axon. $b$, Same field as $a 20 \mathrm{~min}$ after application of okadaic acid $(2 \mu \mathrm{M})$.

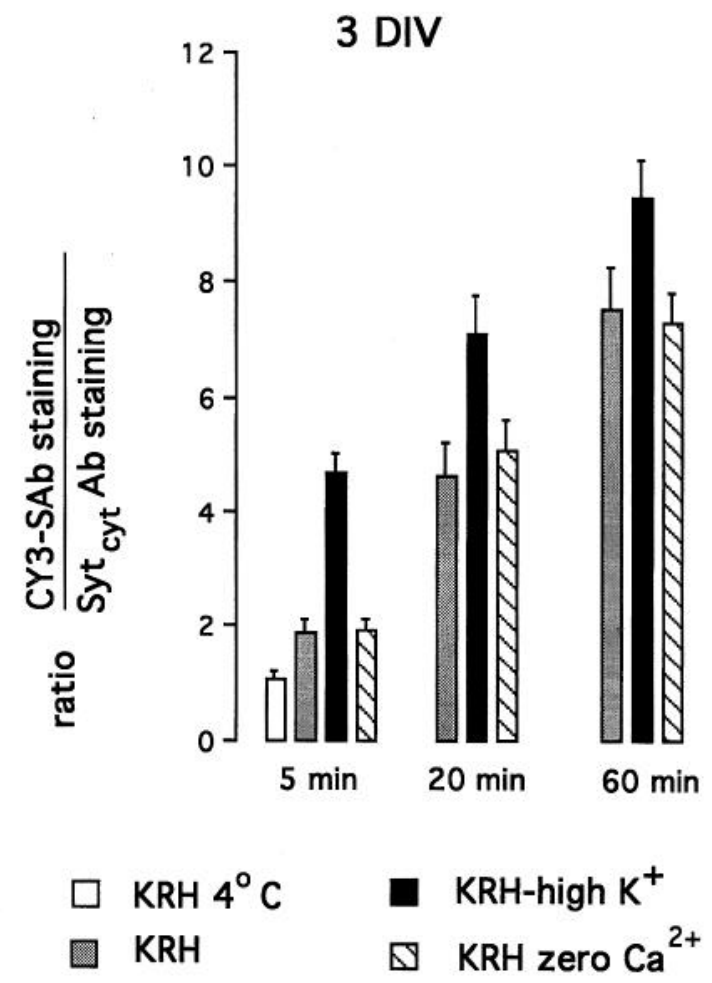

Figure 12. Quantitative analysis of CY3-Syt $\mathrm{C}_{1}$-Abs labeling in axons of isolated (3 DIV) neurons. Cultures were incubated for the times indicated with $\mathrm{CY} 3-\mathrm{Syt}_{1}-\mathrm{Abs}$ in $\mathrm{KRH}, \mathrm{KRH}$-high $\mathrm{K}^{+}, \mathrm{KRH}$-zero $\mathrm{Ca}^{2+}$, and $\mathrm{KRH}$ at $4^{\circ} \mathrm{C}$. After a brief wash cells were then fixed, permeabilized and counterstained with $\mathrm{Syt}_{\mathrm{c}}-\mathrm{Ab}$ (fluorescein). Images of hot spots of immunoreactivity were collected with a CCD camera. Bars represent ratios between the $\mathrm{CY} 3$ and fluorescein fluorescence. Error bars represent SE.
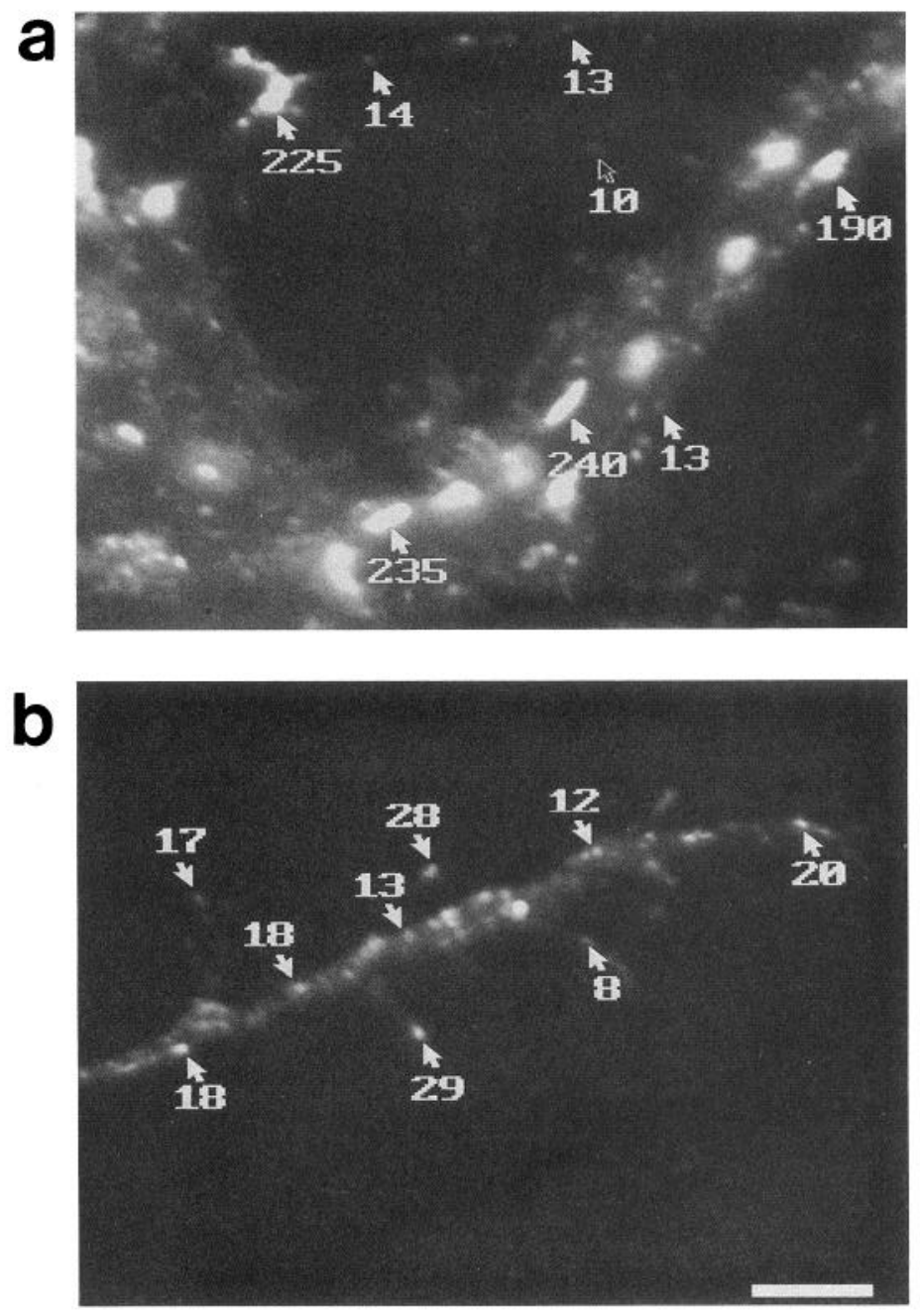

Figure 13. Number of elementary fluorescence units on CY3-Syt - Abs labeled structures. Neurons from a mature culture (8 DIV) $(a)$ and a young culture (3 DIV) $(b)$ were incubated with CY3-Syt - Abs for 20 $\min$ in $\mathrm{KRH}-$ high $\mathrm{K}^{+}$. The level of fluorescence was measured with a CCD camera and the number of elementary units of fluorescence contained in each spot was calculated as described in Materials and Methods. In the field shown in $a$ large structures represent presynaptic clusters and small structures are motile elements primarily localized in axons. Scale bar, $5 \mu \mathrm{m}$.

of isolated SVs. Motile SV clusters of isolated neurons appear to be fundamentally similar to presynaptic clusters. Presynaptic clusters labeled by FM1-43 were shown by video microscopy to merge with each other or split (Betz et al., 1992b) and we show here that the small SV clusters of isolated axons have the same properties. Large presynaptic clusters are disrupted by okadaic acid (Betz and Henkel, 1994), and we report in this study that the same is true for small clusters in isolated axons.

Second, we have found that SV clusters, although present throughout most of the distal axon, are excluded from the leading edge of the growth cone, indicating that SVs exocytosis does not participate either in the elongation of the axon, or in signaling at the axon tip. Presence of SV packages in axonal filopodia which emerge from the preterminal axon shaft suggests that these filopodia may play a role in the formation of synaptic contacts. Thin filopodia, such as those found by us to contain motile SV clusters, were seen in slices of rat hippocampi labeled 
by DiO and observed by confocal microscopy (Dailey et al., 1994). The active motility of SV clusters within these filopodia is likely to be accounted for by microtubulc-bascd motors (Malik and Vale, 1990): presence of CY3-Syt $-\mathrm{Ab}$ labeled particles in the filopodia always correlated with the presence of at least one microtubule (this study) and motility was blocked by microtubule disrupting agents (not shown).

Third, CY3-Syt - Abs revealed that SVs exocytosis is depolarization and $\mathrm{Ca}^{2+}$ dependent even in isolated axons. Such a finding has recently been corroborated by a demonstration of $\mathrm{Ca}^{2}$ dependent glutamate release from isolated neurons prior to the formation of synaptic contacts (Verderio et al., 1995). Thus, the $\mathrm{Ca}^{2+}$ dependence of neurotransmitter release observed at synapses is an intrinsic property of release mediated by SVs and is not dependent upon a structural organization specific of synapses. This finding strengthens the hypothesis that the $\mathrm{Ca}^{2+}$ sensor(s) for the regulated exocytosis of SV is localized on SVs rather than on a docking site at the plasmalemma (Geppert et al., 1994). Our data, however, suggest the occurrence of a higher rate of constitutive SV exocytosis in isolated axons. As we have previously proposed, the formation of synapses may correlate with a down-regulation of basal SV exocytosis (Matteoli et al., 1992). Synapse formation may coincide with a the strengthening of a mechanism which blocks the constitutive exocytosis of SVs and whose action is impaired by cytosolic $\mathrm{Ca}^{2+}$ (Debello et al., 1993; Popov and Poo, 1993). It is of interest that release of glutamate from hippocampal neurons in culture before and after synapse formation was found to be differentially affected by distinct types of $\mathrm{Ca}^{2+}$ channel antagonists (C. Verderio, S. Coco, G. Fumagalli, and M. Matteoli, unpublished observations).

In conclusion, this study describes a new way to quantify SV exocytosis independent of neurotransmitter secretion and contributes new information on the properties of SVs at developmental stages which precede synapse formation. The fundamental molecular mechanisms responsible for the clustering of SVS and for their $\mathrm{Ca}^{2+}$-dependent exocytosis appear to be already in place as the axon advances in search of its target. Formation of a presynaptic compartment may therefore result from the recruitment of preassembled SV functional units at sites of cell contacts. A parallel can be identified with the sequence of events found to occur postsynaptically at the best characterized synapse: the ncuromuscular junction. Formation of small clusters of ACh receptors is an intrinsic property of the postsynaptic cells, while the recruitment of these microclusters to form large postsynaptic aggregates is triggered by the presynaptic element (Hall and Sanes, 1993).

\section{References}

Bartlett WP, Banker GA (1984) An electron microscopic study of the development of axon and dendrites by hippocampal neurons in culture. I. Cells which develop without intracellular contacts. J Neurosci 4:1944-1953.

Bennett MK, Scheller RH (1994) A molecular description of synaptic vesicles membrane trafficking. Annu Rev Biochem 63:63-100.

Betz WJ, Bewick GS (1992) Optical analysis of synaptic vesicle recycling at the frog neuromuscular junction. Science 255:200-203.

Betz WJ, Bewick GS (1993) Optical monitoring of transmitter release and synaptic vesicle recycling at frog neuromuscular junction. J Physiol (Lond) 460:287-309

Betz WJ, Henkel AW (1994) Okadaic acid disrupts clusters of synaptic vesicles in frog motor nerve terminals. J Cell Biol 124:843-854.

Betz WJ, Mao F, Bewick GS (1992a) Activity dependent fluorescent staining and destaining of living vertebrate motor nerve terminals. $J$ Neurosci 12:363-375.

Betz WJ, Bewick GS, Ridge RM (1992b) Intracellular movements of fluorescently labeled synaptic vesicles in frog motor nerve terminals during nerve stimulation. Neuron 9:805-813.

Bialojan C, Takai A (1988) Inhibitory effect of a marine sponge toxin, okadaic acid, on protein phosphatases. Biochem J 256:283-290

Brose N, Petrenko AG, Sudhof TC, Jahn R (1992) Synaptotagmin a calcium sensor on the synaptic vesicle surface. Science 256:10211025.

Ceccarelli B, Hurblut WP, Mauro A (1973) Turnover of transmitter and synaptic vesicles at the frog neuromuscular junction. J Cell Biol 57: 499-524.

Dailey ME, Buchanan J, Bergles DE, Smith SJ (1994) Mossy fiber growth and synaptogenesis in rat hippocampal slices in vitro. J Neurosci $14: 1060-1078$.

DeBello WM, Betz H, Augustine GJ (1993) Synaptotagmin and neurotransmitter release. Cell 74:947-950.

De Camilli P, Jahn R (1990) Pathways to regulated exocytosis in neurons. Annu Rev Physiol 52:625-645.

De Camilli P, Harris SM, Huttner WB, Greengard P (1983) Synapsin I (Protein I), a nerve terminal-specific phosphoprotein. II. Its specific association with synaptic vesicles demonstrated by immunocytochemistry in agarose-embedded synaptosomes. J Cell Biol 96:13551373.

Dotti CG, Sullivan CA, Banker GG (1988) 'The establishment of polarity by hippocampal neurons in culture. J Neurosci 8:1454-1468.

Fesce R, Grohovaz F, Valtorta F, Meldolesi J (1994) Neurotransmitter release: fusion or 'kiss-and-run'? Trends Cell Biol 4:1-4.

Fletcher TL, Cameron P, De Camilli P, Banker G (1991) The distribution of synapsin I and synaptophysin in hippocampal neurons developing in culture. J Neurosci 11:1617-1626.

Forscher P, Smith SJ (1988) Actions of cytochalasins on the organization of actin filaments and microtubules in a neuronal growlh cone. J Cell Biol 107:1505-1516.

Geppert M, Goda Y, Hammer RE, Li C, Rosahl TW, Stevens CF, Sudhof TC (1994) Synaptotagmin I: a major $\mathrm{Ca}^{2+}$ sensor for transmitter release at a central synapse. Cell 79:717-727.

Hall ZW, Sanes JR (1993) Synaptic structure and development: the neuromuscular junction. Cell [Suppl] 72:99-121.

Harris KM, Stevens JK (1989) Dendritic spines of CA 1 pyramidal cells in the rat hippocampus: serial electron microscopy with reference to their biophysical characteristics. J Neurosci 9:2982-1997.

Heuser JE, Reese TS (1973) Evidence for recycling of synaptic vesicle membrane during transmitter release at the frog neuromuscular junction. J Cell Biol 57:315-344.

Jessell TM, Kandell ER (1992) Synaptic transmission: a bidirectional and self-modifiable form of cell-cell communication. Cell [Suppl] 72:1-30.

Liley A (1956) The effects of presynaptic polarization on the spontaneous activity at the mammalian neuromuscular junction. J Physiol (Lond) 134:427-443

Malik F, Vale R (1990) A new direction for kinesin. Nature 317:713 714.

Matteoli M, Takei K, Perin MS, Sudhof TC, De Camilli P (1992) Exoendocytotic recycling of synaptic vesicles in developing processes of cultured hippocampal neurons. J Cell Biol 117:849-861.

Montecucco C, Schiavo G (1993) Tetanus and botulism neurotoxins: a new group of zinc proteases. Trends Biochem Sci 18:324-327.

Mundigl O, Matteoli M, Daniell L, Thomas-Reetz A, Metcalf A, Jahn R, De Camilli P (1993) Synaptic vesicles proteins in early endosomes in cultured hippocampal neurons: differential effects of brefeldin A in axon and dendrites. J Cell Biol 122:1207-1221.

Niemann H. (1991) Molecular biology of clostridial neurotoxins. In: Sourcebook of bacterial protein toxins (Alour JE, Freer JH, eds), pp 303-348. Orlando, FL: Academic.

Parton RG, Simons K, Dotti CG (1992) Axonal and dendritic endocytic pathway in cultured neurons. J Cell Biol 119:123-139.

Popov SV, Poo MM (1993) Synaptotagmin: a calcium-sensitive inhibitor of exocytosis? Cell 73:1247-1249.

Ryan TA, Reuter H, Wendland B, Schweizer FE, Tsien RW, Smith SJ (1993) The kinetics of synaptic vesicle recycling measured at a single presynaptic boutons. Neuron 11:713-724.

Slot JW, Geuze HJ (1983) A new method of preparing gold probes for multiple-labcling cytochcmistry. Eur J Cell Biol 38:87-93.

Southwick PL, Ernst LA, Tauriello EW, Parker SR, Mujumdar RB, Mujumdar SR, Clever HA, Waggoner AS (1990) Cyanine dye labeling 
reagents-carboxymethylindocyanine succinimidyl esters. Cytometry 11:418-430.

Sudhof TC, De Camilli P, Niemann H, Jahn R (1993) Membrane fusion machinery: insights from synaptic proteins. Cell 75:1-4.

Torri-Tarelli R, Villa A, Valtorta F, De Camilli P, Greengard P, Ceccarelli B (1990) Redistribution of synaptophysin and synapsin I during $\alpha$-latrotoxin-induced release of neurotransmitter at the neuromuscular junction. J Cell Biol 110:449-460.

Vale RD (1987) Intracellular transport using microtubule-based motors. Annu Rev Cell Biology 3:347-378,
Verderio C, Coco S, Fumagalli G, Matteoli M (1995) Calcium-dependent glutamate release during neuronal development and synaptogenesis: different involvement of $\omega$-AgaIV A and $\omega$-CTx-GVIA sensitive channels. Proc Natl Acad Sci USA, in press.

Wendland B, Miller KG, Schilling J, Scheller RH (1991) Differential expression of the p65 gene family. Neuron 6:993-1007.

Zucker RS, Delaney KR, Mulkey R, Tank DW (1991) Presynaptic calcium in transmitter release and posttetanic potentiation. Ann NY Acad Sci 635:191-207. 